\title{
Simulation Validation of Static and Forced Motion Flow Physics of a Canard Configured TransCruiser
}

\author{
Mehdi Ghoreyshi ${ }^{1}$, Ramy Korkis-Kanaan, Adam Jirásek, \\ Russell M. Cummings, and Andrew J. Lofthouse \\ High Performance Computing Research Center, U.S. Air Force Academy \\ USAF Academy, Colorado 80840-6400, USA
}

\begin{abstract}
The basic objective of this work is to validate CFD simulations performed on a transonic cruiser configuration called the TCR. The low speed aerodynamic characteristics of the TCR have previously been investigated at the Russian TsAGI T-103 wind tunnel as part of the European Framework 6 SimSAC project. The experimental results showed that static and dynamic pitch moment curves are very nonlinear. These experimental data are used in this work to validate CFD predictions using the Cobalt flow solver with an overset grid approach. Two types of wind tunnel tests were conducted: static tests for angle-of-attack sweeps at zero degrees sideslip and angle-of-sideslip sweeps at different angles of attack. The dynamic tests include forced sinusoidal oscillations in one of three modes of pitch, yaw, and roll. Both static and dynamic tests were conducted with/without a vertical tail and at different canard deflections. Dynamic tests are small- and large-amplitude motions with frequencies of $0.5,1.0$, and $1.5 \mathrm{~Hz}$. CFD results were obtained with different turbulence models and using a single mesh or an overset grid approach, and then compared with the experimental data. The effects of the canard downwash flow on the wing aerodynamic performance are also investigated. The comparison between the experimental and CFD simulations show that the results match well. The overset mesh that includes a gap between the canard and fuselage leads to the same predictions as the single mesh that has no gaps. The CFD solutions show that vortices are formed over the canard, fuselage, LEX, wing, and the vertical tail (at sideslip angles). Each vortex appears to have a primary vortex accompanied by a smaller counter-rotating secondary vortex. These vortices are influenced by the canard presence and deflection. At high angles of attack, the canard vortex has two favorable effects in terms of increasing the maximum lift and delaying the wing vortex breakdown. In the range of angles of attack between $18^{\circ}$ to $24^{\circ}$, the canard vortex core moves upward off the canard surface and the LEX and wing vortices interact and then merge; both effects lead to a sudden change in the slopes of the force and moment curves. Finally, the CFD data show that increasing the canard deflection produces a stronger vortex over the canard, but leads to smaller fuselage and LEX vortices.
\end{abstract}

Keywords:

Transonic Passenger Aircraft, Overset mesh, CFD, Canard-Wing interaction 


\section{Nomenclature}

$\begin{array}{ll}A_{0} & \text { motion amplitude, rad } \\ a & \text { speed of sound, } \mathrm{m} / \mathrm{s} \\ b & \text { wing span, } \mathrm{m} \\ C_{D} & \text { drag coefficient, } D / q_{\infty} S \\ C_{N} & \text { normal-force coefficient, } N / q_{\infty} S \\ C_{M x} & \text { roll moment coefficient, } M_{x} / q_{\infty} S b \\ C_{M y} & \text { pitch moment coefficient, } M_{y} / q_{\infty} S c \\ C_{M z} & \text { yaw moment coefficient, } M_{x} / q_{\infty} S b \\ C_{p} & \text { pressure coefficient, }\left(p-p_{\infty}\right) / q_{\infty} \\ C_{Y} & \text { side-force coefficient, } Y / q_{\infty} S \\ c & \text { mean aerodynamic chord, } \mathrm{m} \\ D & \text { drag force, } \mathrm{N} \\ f & \text { frequency, Hz } \\ N & \text { normal force, } \mathrm{N} \\ M_{x} & \text { roll moment, } \mathrm{N}-\mathrm{m} \\ M_{y} & \text { pitch moment, } \mathrm{N}-\mathrm{m} \\ M_{z} & \text { yaw moment, } \mathrm{N}-\mathrm{m} \\ M & \text { Mach number, } \mathrm{V} / \mathrm{a} \\ p & \text { static pressure, Pa } \\ p_{\infty} & \text { freestream pressure, Pa } \\ q_{\infty} & \text { dynamic pressure, Pa, } \rho V^{2} / 2 \\ R e & \text { Reynolds number, } \rho V c / \mu \\ S & \text { Planform area, } \mathrm{m}^{3} \\ t^{*} & \text { non-dimensional time step, } V t / c \\ t & \text { time, s } \\ V & \text { freestream velocity, } \mathrm{m} / \mathrm{s} \\ x, y, z & \text { aircraft position coordinates }\end{array}$

\section{Greek}

$\alpha$

angle of attack, rad

$\dot{\alpha} \quad$ time-rate of change of angle of attack, $\mathrm{rad} / \mathrm{s}$

Email address: Mehdi.Ghoreyshi@usafa.edu (Mehdi Ghoreyshi)

${ }^{1}$ Corresponding Author.

"The views expressed in this paper are those of the author and do not reflect the official policy or position of the United States Air Force, Department of Defense, or the U.S. Government." Distribution A. Approved for Public Release. Distribution unlimited. 


\section{Subscripts}

$\begin{array}{ll}c & \text { canard } \\ w & \text { wing }\end{array}$

side-slip angle, rad

control surface deflection, rad

air density, $\mathrm{kg} / \mathrm{m}^{3}$

air viscosity, $\mathrm{kg} /(\mathrm{m} . \mathrm{s})$

\section{Introduction}

Computational fluid dynamics(CFD) simulations are anticipated to become the primary tool in the design of modern commercial and military aircraft. The traditional handbook methods and low-fidelity aerodynamic tools fail to predict accurately the aerodynamic behavior over an extended flight envelope and/or of a novel configuration [1]. Flight tests are expensive, sometimes require risky maneuvers, and become available late in the design cycle. As a result, CFD predictions and wind tunnel experiments play a crucial role in the development of modern aircraft prior to fabrication. Specifically, CFD has become reliable enough to detect the source of undesirable flight characteristics experienced in the flight testing [2]. This ensures the early availability of high quality aerodynamic models for design of the control system and minimizes risk and uncertainty in a new airplane product. However, CFD techniques are subject to uncertainties due to the choice of numerical and geometrical models, and therefore CFD simulations must be validated and evaluated on the basis of experimental data. This work considers the application of CFD modeling to predict static and forced motion aerodynamic responses of a canard configured TransCRuiser named the TCR.

The TCR (shown in Figure 1 (a)) is a conceptual design of a civil transport aircraft operating at transonic speeds, with a cruise Mach number of 0.97 [3]. The design was proposed by the Swedish aerospace company SAAB; the initial concept was examined and modified within the European Framework 6 SimSAC project [4]. The final TCR aircraft is a canard-wing-vertical tail configuration and includes a wing leading edge extension (LEX). The canard is an all moving surface and a close-coupled type. Both the wing and canard are swept back $60^{\circ}$ causing vortex formation at moderate to high angles of attack. The transonic design speed was chosen to highlight the shortcomings of the handbook methods and linear aerodynamic codes available in the SimSAC project. Even at low subsonic speeds, the aircraft has very nonlinear aerodynamic behavior due to canard/wing interference effects $[1,5,6]$. The presence and deflection of the canard will affect the wing performance, specifically for a close-coupled canard configuration [7]. As a result, the TCR aircraft provides a challenging task for CFD simulation.

In this work, computational predictions are compared with available wind tunnel measurements of the SimSAC project to evaluate the accuracy of standard CFD models and 
methods. The wind tunnel model was built at Politecnico di Milano in Italy and then tested in the TsAGI T103 wind tunnel in Russia at low speeds and up to high angles of attack. Several configurations were investigated to allow consideration of the influence of single components (vertical tail and canard) on the overall performances. The static tests included a variation of side-slip angles, angles of attack ranging from $-10^{\circ}$ to $40^{\circ}$ with step of $2^{\circ}$, and the canard deflections from $-30^{\circ}$ to $10^{\circ}$ with step of $5^{\circ}$ and an asymmetric deflection with 10/ +10 degrees. Dynamic tests include small- and large-amplitude motions with frequencies of $0.5,1.0$, and $1.5 \mathrm{~Hz}$ and were tested in pitch, roll, and yaw modes. For small amplitude motions, dynamic derivatives were estimated using a linear regression method and included in the experimental data [8]. In addition, the TCR was tested for plunged forced oscillations to measure $\dot{\alpha}$ derivatives.

In the experiments, the left and right canard panels were deflected to eight different positions including an asymmetric position. Regeneration of the mesh for all these cases can be a very time-consuming and labor-intensive task. This work investigates the use of an overset method for modeling the TCR canard deflection. In an overset method, independent meshes are generated around different body elements and then the governing flow equations are solved independently for each mesh. Information is transferred between these meshes using an interpolation technique. Since these meshes are created for each component and they can be moved/rotated independently large displacements of control surfaces are possible without the regeneration of the mesh. The results of the overset mesh of this work are compared with experimental data and those predicted by a single mesh at a canard angle of zero degrees. This allows investigation of the effects on the aerodynamic predictions of the gap that exists between the canard and fuselage of the overset meshes.

The computational results of this work will aid in the understanding of the flowfield over the TCR. Specifically, the TCR wind tunnel experiments only provide total integrated forces and moments; surface pressure measurements and flowfield information are not available. In addition, the load balance used in the wind tunnel could not measure the drag force. The surface flow conditions are easily available from the CFD simulations; forces and moments can also be isolated for each surface or region of interest to study the canard/wing interference. Note that it took about three years to plan the wing tunnel experiments of the TCR, build the model, and complete all the tests.

There are three objectives in the present study: 1) to contribute to the understanding of the flow physics of the TCR configuration, 2) to develop validated aerodynamic models for the TCR using an overset grid method 3) and to investigate the effects of the canard downwash flow on the wing aerodynamic performance. The work is organized as follows. The first section reviews the canard-wing interaction effects. Next, a basic introduction of the CFD flow solver is provided. The TCR geometry and mission requirements are then detailed. The computational grids are presented in the following section. The results are then presented and discussed, followed by concluding remarks. 


\section{Canard-Wing Interaction}

A canard-wing configuration is an attractive design choice for a high-speed, highly maneuverable aircraft [9]. The presence of a vortex generating surface ahead of the wing increases the maximum lift, reduces the trim drag, delays the wing stall, and improves the maneuverability [10]. While a conventional aircraft should generate some negative lift on the tail to balance the wing pitch moment around center of gravity, the canard will produce a possible upward lift. The main wing of a conventional aircraft must compensate the tail negative lift, causing the induced and trim drag to increase as result of increased lift [11]. A canard offers two possible benefits with reducing the wave drag: first, it improves the longitudinal variation of the cross-sectional area, particularly for a close-coupled canard [10], and second, it makes an aerodynamically clean fuselage aft section compared to a tailed configuration [12].

A canard has many benefits for a transonic cruiser. A properly designed and placed canard will stall before the main wing, and as a result, the airplane nose drops (an uncommanded pitch) which prevents the main wing from entering the stall regime. In addition, the canard provides a positive pitch control which could counteract the nose down tendency of the airplane as it enters the transonic regime("Mach tuck"). Finally, the canard improves the stall/spin recovery. However, the canard is a statically destabilizing device because of its location ahead of the center of gravity [13]. Therefore, the canard size should be as small as possible to minimize its destabilizing effect, but, this decreases the pitch control effectiveness as well [11].

Canard-wing configurations can be classified into long-coupled and close-coupled as shown in Figure 2. Roskam [14] suggested the ratio between the distance between canard (mean aerodynamic center) and the wing (mean aerodynamic center) to the mean aerodynamic chord to classify airplanes into long-coupled and close-coupled types; a long-coupled design has a ratio greater than five, a short-coupled typically has a ratio less than three.

If the design focus is on reducing aircraft drag at cruise conditions, the canard should be placed far ahead of the wing. This configuration minimizes the canard-wing interference effects and requires a smaller upward load on the canard; both effects would help to reduce the trim drag [9]. One example is the $\mathrm{X}-31$, which has canards that deflect between +20 and -70deg for pitch control requirements [15]. The problems related to a long-coupled configuration are mainly stability issues. Torenbeek [9] stated that the size of a long-coupled canard should be less than $10 \%$ of the main wing to achieve stability. This makes a longcoupled canard the primary pitch control surface rather than a lifting surface. Therefore a long-coupled canard typically requires large deflections for the pitch control.

The canard/wing interaction for a short-coupled canard increases the maximum lift and drag in comparison to a long-coupled canard. Examples of the close-coupled configurations are NASA X-29, SAAB Viggen and Grippen, and European Fighter Aircraft. Typically, close-coupled canards are bigger than long-coupled ones, and therefore they are lifting surfaces as well as a control surface [16]. These highly-loaded surfaces will produce excessive drag as well. At small angles of attack, the canard induces a downwash over the wing within the canard span and an upwash outside its span $[17,18,16]$. As a result, while the flow 
tends to remain attached over the wing behind the canard, it tends to separate outside the canard [16]. Although, this leads to a loss of lift on the wing compared with a wing only configuration, the overall aircraft lift will be identical to a canard-off configuration due to the increased lift by the canard. Oleary [16] stated that "at low angles of attack $\left(<10^{\circ}\right)$ the lift-curve slope of a model with or without a canard is identical."

For slender delta canard/wing surfaces, the flow at moderate to high angles of attack is characterized by two large counter-rotating primary vortices formed over the upper surface. The sharp leading edges of slender wings cause the boundary layer to separate and the separated shear layers to roll up into vortices. The shear layer may exhibit instabilities that increase the vortical substructures and, therefore, the vortices increase in both size and strength as they extend downstream. A close-coupled canard has favorable interference between the canard and wing leading-edge vortices [16]. In these configurations, the canard vortex helps to delay the wing vortex breakdown to higher angles compared with a wing only configuration. As a result, a canard-on delta wing has a larger maximum lift coefficient than a canard-off configuration [19].

\section{CFD Solver}

The flow solver used for this study is the Cobalt code [20] that solves the unsteady, threedimensional and compressible Navier-Stokes equations in an inertial reference frame. The ideal gas law and Sutherland's law close the system of equations and the entire equation set is nondimensionalized by free stream density and speed of sound [20]. The Navier-Stokes equations are discretised on arbitrary grid topologies using a cell-centered finite volume method. Second-order accuracy in space is achieved using the exact Riemann solver of Gottlieb and Groth [21], and least squares gradient calculations using QR factorization. To accelerate the solution of the discretized system, a point-implicit method using analytic firstorder inviscid and viscous Jacobians is implemented. A Newtonian sub-iteration method is used to improve time accuracy of the point-implicit method. Tomaro et al. [22] converted the code from explicit to implicit, enabling Courant-Friedrichs-Lewy numbers as high as $10^{6}$. Some available turbulence models are the Spalart-Allmaras model [23], Wilcox's k- $\omega$ model [24], and Mentor's SST model [25].

Cobalt includes an overset mesh method that allows the independent translation and rotation of each grid around a fixed or moving hinge line. In this method, overlapping grids are generated individually, without the need to force grid point alignement with neighboring components [26]. In Cobalt, the overlapping grids are treated as a single mesh using a grid-assembly process. This includes a hole-cutting procedure in overlapping regions and interpolation between overlapping grids. The translation and rotation of overset grids around the hinge line are input to the code using a Grid Control File (GCF). The hinge line is defined by a reference point and a vector combination. The rotations are based on the right-hand rule and consist of angles in an order of pitch, yaw, and roll angle. These angles are estimated from the deflection angle of a control surface and the relative angles between the hinge line and grid coordinate axes. 


\section{Test Case}

The TCR, developed by SAAB, is a conceptual design of a civil transport aircraft operating at transonic speeds. The concept features a low wetted area, fuel efficiency at transonic cruise, low noise radiation, relaxed static stability boundaries, and low maneuver and trim drag [8]. The design specifications are detailed by Eliasson et al. [5], with some examples given below:

Payload: Nominal design for 200 passenger in economy class

Design Cruise Speed: $M_{D}=0.97$ at an altitude at or above $37,000 \mathrm{ft}$.

Range: $5,500 \mathrm{~nm}$, followed by $250 \mathrm{~nm}$ flight to an alternate airfield and 0.5 hour loiter time at an altitude of $1,500 \mathrm{ft}$.

Take-off and landing: Take-off distance of $8858 \mathrm{ft}$ at an altitude of 2,000 ft, ISA +15 and maximum take-off weight. Landing distance of $6561 \mathrm{ft}$ at an altitude of 2,000 ft, ISA and maximum landing weight with maximum payload and normal reserves.

Power plants: Two turbofans

The initial concept by SAAB was a conventional tailed configuration. The initial design study revealed that a very large horizontal tail deflection should be applied to trim the aircraft at the design point [1]. The initial design was then investigated further within the SimSAC projects to improve the aircraft stability and control characteristics; the final design became a canard-wing-vertical tail configuration as shown in Figure 1 (a).

The TCR canard is an all-moveable control surface and the primary pitch control device for the aircraft. The canard exposed area is about 15 percent of the wing reference area. The apex positions of the wing and canard are at $26 \%$ and $12 \%$ of the fuselage length [1], making the canard a close-coupled type.

A wind tunnel model was designed next and built to one-fortieth scale by Politecnico di Milano. The wind tunnel model layout and dimensions are shown in Figure 3. TCR airfoil information is not readily available, but, the canard uses a symmetric airfoil design. The wing airfoil section at $Y=0.3 \mathrm{~m}$ as extracted from the CAD is shown in Figure 4. Other geometry parameters are given in Table 1.

Note that the TCR main wing has a round (blunt) leading edge. The vortical flow behavior over slender wings with a blunt leading edge is very different from those of sharp leading edges [27, 28]. The vortex separation location is constant on a sharp leading edge wing, but for blunt wings, the vortex flow structure is very complicated and depends heavily on the leading edge bluntness and the wing sweep angle.

\section{Computational Grids}

Half- and full-geometry meshes were used, corresponding to the TCR wind tunnel model with and without the canard. The RANS meshes were generated in two steps. In the 
Table 1: TCR wind tunnel model parameters

\begin{tabular}{ll}
\hline \hline Parameter & Values \\
\hline Model scale & $1: 40$ \\
Wing area, $S_{w}$ & $0.3056 \mathrm{~m}^{2}$ \\
Canard area, $S_{c}$ & $0.045 \mathrm{~m}^{2}$ \\
Wing span, $b_{w}$ & $1.12 \mathrm{~m}$ \\
Canard span, $b_{c}$ & $0.3 \mathrm{~m}$ \\
Mean aerodynamic chord, $c$ & $0.2943 \mathrm{~m}$ \\
CG position form the aircraft nose, $x_{C G}$ & $0.87475 \mathrm{~m}$ \\
Total fuselage length & $1.597 \mathrm{~m}$ \\
Fuselage Diameter & $0.925 \mathrm{~m}$ \\
\hline \hline
\end{tabular}

first step, the inviscid tetrahedral mesh was generated from a clean configuration using the ICEMCFD code. The inviscid mesh was then used as a background mesh by TRITET[29, 30] which builds prism layers using a frontal technique. TRITET rebuilds the inviscid mesh while respecting the size of the original inviscid mesh from ICEMCFD. Symmetric meshes are shown in Figure 5 for the TCR model with and without canard. The symmetric grid without the canard has 26 Million cells and the mesh with the canard contains 33 million cells. A finer mesh having around 33 Million cells was also generated for the TCR without the canard.

The average $y+$ for these meshes is 0.281 . Figure 6 shows the $y+$ values of the single mesh with non-deflected canard at zero degrees angle of attack. In addition, Cobalt checks the grid quality and reports a score; this score is directly related to a particular part of the second-order accurate spatial operator inside Cobalt. The reported score is averaged over all the cells and ranges from zero to hundred, such that the lower the grid score, the more numerical dissipation is added to the solution [20]. Cobalt reports an average grid score of 93 for the meshes of this work.

Wind tunnel experiments were run for the canard deflection angles ranging from $-30^{\circ}$ to $10^{\circ}$ with a step of $5^{\circ}$. An overset grid approach is used in this work for simulating the canard deflections. The overlapping grids were generated individually for the body and canard, without the need to force grid points aligned with neighboring components. However, a grid assembly approach requires some gaps between the canard and the body. The grid also needs to be refined around the gaps which often make the grid size larger than a single grid. In this work, two hybrid RANS meshes were generated from the half and full geometry models of the TCR without canard surfaces. The grids for left and right canards were generated separately and overset onto the main grid. The background and minor overset grids were also generated using the ICEMCFD and TRITET codes. The assembled grid of the half geometry has about 40 million cells; the overset grid at a canard deflection of $-30^{\circ}$ is shown in Figure 7. 


\section{Experimental Setup}

The TCR wind-tunnel model was built with a geometric scaling factor of 1:40 of the actual size to fit the size requirements of the TsAGI subsonic wind tunnel. This wind tunnel has an open jet working section of the continuous type with an elliptical cross section of 4.0 $\times 2.33 \mathrm{~m}[8]$. The wind tunnel can operate at velocities up to $80 \mathrm{~m} / \mathrm{s}$ and Reynolds numbers up to 5.5 million. For the dynamic tests, the model was mounted on a platform driven by actuators. The model center of gravity was located along the fuselage center line at $54.78 \%$ of the total length from the foremost point. The moment reference point and the center of oscillatory motion coincide with this point. Figure 1 (b) shows the TCR wind tunnel model at the TsAGI subsonic wind tunnel; note that there is a gap region between the canard and the fuselage.

The normal and lateral forces and the moment coefficients from static and large amplitude pitch oscillations were measured. The mean values, in-phase and out-of-phase components of the force and moment coefficients were also measured from the rotary and oscillatory motions. All aerodynamic coefficients are given in the body axis system. The coordinate system used as well as forces and moments are shown in Figure 8.

All experiments were run at a free-stream speed of $40 \mathrm{~m} / \mathrm{s}$, which corresponds to a sea level Mach number of 0.117 , and a Reynolds number of 0.778 million based on the mean aerodynamic chord of the wind tunnel model. These experimental data can be obtained from the SimSAC project website (http://www.ceasiom.com) and are used in this work to validate the overset grid approach and the Cobalt CFD solver used at the U.S. Air Force Academy (USAFA).

\section{Results}

In all subsequent simulations, the free-stream velocity is $40 \mathrm{~m} / \mathrm{s}$ and the Reynolds number corresponds to 0.778 million based on the mean aerodynamic chord. All simulations were run on the Department of Defense High Performance Computing Modernization Program (HPCMP).

All aerodynamic coefficients in this work (except drag) are given in the body axis system, as with the experimental data. Drag coefficients are only calculated for cases with zero sideslip angles and are defined in the wind axis. The simulation tests included side-slip angle sweeps from $-16^{\circ}$ to $16^{\circ}$ with steps of $4^{\circ}$ and angle-of-attack sweeps ranging from $0^{\circ}$ to $40^{\circ}$ with steps of $2^{\circ}$.

\subsection{Static Results}

The turbulence modeling and grid refinement results are briefly described first. Figure 9 compares CFD predictions with the experimental data of the TCR without canard using a single grid (medium size mesh). CFD data are obtained with the Spalart-Allmaras (SA) [23] and the SA with Rotation Correction (SARC) turbulence modelk [31]. The results show that the SARC model matches the experimental results better than the SA model, particularly 
at high angles of attack. Specifically, the SARC model has been successfully used in aerodynamic prediction of aircraft with vortical flows, at least before the appearance of vortex breakdown. The SARC model is therefore used for all subsequent simulations.

In addition, the grid refinement results are shown in Figure 10 for the TCR without canard using single grids with 26 and 33 million cells. Figure 10 shows that the predictions using the medium grid converged to those predicted by the fine grid. The medium-size mesh is therefore used in all subsequent simulations.

CFD validation results are shown in Figure 11 for the angle-of-attack sweeps at zero degrees of sideslip. The CFD simulations were performed on the single grids with and without the canard; in the former, the canard is not deflected, i.e. $\phi_{c}=0^{\circ}$. All solutions were run up to 4,000 iterations, achieving a density residual drop of three orders of magnitude. Normal force, pitch moment, and drag force were time-averaged over the last 500 iterations and were compared with the TCR experimental data in Figure 11. Note that the drag force was not measured in the wind tunnel experiments.

Figure 11 shows very good agreement between Cobalt predictions and the experimental results for both canard on and off configurations. For angles of attack less than eight degrees, the canard presence has no significant effects on the aircraft normal force (lift) and drag. At higher angles, however, the canard-configured TCR shows higher normal force and drag values. The pitch moment curves have different slopes for the canard on and off geometries; the pitch moment values only match at zero degrees of angle of attack and are different elsewhere. The canard is a destabilizing device and causes a less negative (or more positive) total pitch moment as shown in Figure 11(b).

Two breaks can be seen in the experimental pitch moment plots of Figure 11(b) for the canard-on configuration. These breaks were also reported and detailed by Eliasson et al. [5]. The breaks occur at angles of about $\alpha=8^{\circ}$ and $\alpha=20^{\circ}$; while in the first break the pitch moment begins to increase, the pitch moment suddenly drops in the second break and then continues to grow again [5]. Figure 11(b) shows that CFD accurately predicts the first break but the second break is slightly off.

A detailed assessment of the plots of Figure 11 show that dips can be observed in the CFD normal-force coefficients for the canard on and off configurations; the dips are located at angles of attack between $18^{\circ}$ to $24^{\circ}$; the dip becomes smaller in the presence of the canard. Small dips can also be seen in the drag force plots at the same angles. The pitch moment slope of the canard-off geometry is negative and then becomes nearly zero for angles above $28^{\circ}$; there is a sudden change in the slope for angles between $18^{\circ}$ to $24^{\circ}$. On the other hand, the pitch moment slope in the presence of the canard becomes positive around $12^{\circ}$; it becomes negative for angles between $20^{\circ}$ to $24^{\circ}$ and again takes positive values, though they are small.

The CFD results of Figure 11 may raise the question as to why CFD shows a dip in the plots but not the experiment? An answer to this question might be found be assessing Figure 11 (d) which shows the CFD normal force of the canard-off geometry for a sweep of angle of attack with different run times. The results show that no dip or a very small dip can be seen in the plots if the solutions only run for 2,000 iterations. The solutions at 2,000 iterations have already reached the steady-state values for most angles, but they still change 
for angles between $18^{\circ}$ to $24^{\circ}$ with more iterations. A dip will be formed in the plots by iterating solutions up to 4,000. More run time, however, do not make much difference from the solutions found at 4,000 iterations. This confirms that solutions at these angles need enough time to converge and stabilize. Unfortunately, the wind tunnel details (time run at each angle) are not available to comment on the time over which the experimental data was taken.

To better understand the canard and wing contributions to total forces and moments, Figure 12 shows the lift, drag, and pitch moment coefficients for individual surfaces. Figure 12(a) shows that at small angles of attack, the wing in the presence of the canard has less slightly lift than a wing-only configuration; this is mainly due to canard downwash effects on the wing. However, the total lift remains the same because of the additional lift generated on the canard. At higher angles the wing behind the canard produces more lift than a wing-only geometry because of the canard vortex effects on the wing. These vortices will increase wing drag as well. Figure 12(a) shows that the canard stalls earlier than the main wing. Dips can again be seen in the wing plots. Figure 12(b) shows that the canard pitch moment slope is positive for all angles, confirming that the canard is a destabilizing surface. The wing pitch moments (with and without canard) match with each other at small angles of attack and have negative slopes for most angles.

Figure 13 details the canardless TCR flow solutions at different angles of attack and zero sideslip angle. For several $x$ positions, slices were created and colored by the vorticity magnitude. The boundary layer thickness is related to the diffusion of vorticity generated on the surface. The slices of Figure 13 become thicker in the streamwise direction as the boundary layer grows. The solutions also show that the boundary layer over the fuselage is much thicker than the wing boundary layer, possibly due to a longer fuselage section and the sharp angle between the fuselage nose cone and fuselage cylindrical section (see Figure 3). Apart from the boundary layer growth, no other feature of interest was found for TCR solutions below $6^{\circ}$ angle of attack.

At $6^{\circ}$ angle of attack, the canardless TCR shows a wing tip vortex starting at about quarter chord of the wing tip. Note also that small vortices are formed over the wing starting at different wing leading edge sections (but do not originate from the wing apex) as shown in Figure 13(a). As the angle of attack increases, these vortices become bigger and stronger, they move inboard and the starting point moves towards the wing apex. At $\alpha=12^{\circ}$, an outboard vortex is formed over the wing, originating from the wing apex. This vortex interacts with the wing tip vortex. Also, an inboard vortex is formed which originates from the LEX. Both of these vortices have a vortex structure containing a primary and a small counter-rotating secondary vortex. These vortices can be seen in Figure 13(b). In addition, the boundary layer over the fuselage separates and rolls into two counter-rotating vortices.

Further increase in the angle of attack makes all vortices stronger. The outboard vortex still emanates from the wing apex and travels downstream, however its core begins to move towards the inboard (LEX) vortex with increased angle of attack. At $\alpha=18^{\circ}$ the inboard and outboard vortices interact and merge downstream as shown in Figure 13(c). Only one wing vortex travels downstream. As the angle of attack increases further, the outboard 
vortex continues to move inboard and the merging point shifts upstream. The vortex core locations and the merge point do not change much after $\alpha=24^{\circ}$. The dips seen in the normal force and drag force of Figure 11 are related to the interaction and merging of vortices at angles between $18^{\circ}$ to $24^{\circ}$. For higher angles, the merged vortex becomes larger but the merging point does not change much. For example, Figs. 13(d)-(e) show the vortices at $24^{\circ}$ and $30^{\circ}$ angles of attack, respectively. These figures show that the vortex core position and the merging point are still the same, however, the vortices have different sizes. At higher angles, the fuselage vortex interacts with the LE vortex as well as the merged vortex. This can be seen in Figure 13(f) for $40^{\circ}$ angle of attack.

Likewise, Figures 14 and 15 detail the canard-configured TCR flow solutions at different angles of attack and zero sideslip angle. Comparing with canardless solutions, Figure 14(a) shows that at $\alpha=6^{\circ}$, the wing flow solutions of the canard on and off configurations are very similar. Figure. 14(a) also shows that a tip vortex is formed over the canard. At $\alpha=12^{\circ}$, the starting point of the canard vortex is at the canard apex. Due to canard downwash and upwash effects, the wing solutions for canard on and off are slightly different for this and higher angles of attack. The wing in the presence of the canard shows smaller inboard and fuselage vortices than the canardless configuration; this is due to canard downwash effects that reduce the local angle of attack behind the canard span. On the other hand, the wing outboard vortex is slightly bigger in the presence of the canard.

Figure 14 shows that the canard vortex becomes larger with increasing angle of attack. At $\alpha=18^{\circ}$, the wing vortices merge, but the inboard vortex is much smaller for the canard-configured than for the canardless geometry. For higher angles, the TCR canard has favorable effects on the wing aerodynamic performance. At $24^{\circ}$ angle of attack, the wing behind the canard has a stronger merged vortex than the wing-only configuration. This can be seen in Figures 14(d) and 15(d); this is mainly due to canard upwash outside the canard span. This leads to more negative pressure region over the wing surface, higher lift, and delayed vortex breakdown. Note that the fuselage vortex is still small for the canard-configured TCR because of downwash effects behind the canard span.

More details of the flows around the canard-configured TCR can be found in Figure 16 which shows predicted spanwise pressure coefficients at different locations over the canard and wing for several angles of attack. In these figures, the corresponding pressure distributions on the upper and lower surfaces of the wing are shown. Figures 16 (b) and (c) show that at $8^{\circ}$ angle of attack, a leading-edge vortex is formed over the canard upper surface which causes a large negative peak in the upper surface pressure coefficients. The canard vortex (and therefore the pressure peak) moves slightly inboard as it moves downstream. This canard vortex is the source of the first break seen in the TCR pitch moment plots.

At $18^{\circ}$ angle of attack, the canard vortex (and hence the pressure peak) can still be seen in the tap-1 location (Figure 16 (b)). This vortex is much larger in size and its core is closer to the fuselage than the vortex seen at $8^{\circ}$ angle of attack. The canard vortex will move upward off the surface as it moves downstream; no sign of any vortex can be seen at tap-2 location as shown in Figure 16 (c). This figure shows no pressure peak due to a leading-edge vortex. At $24^{\circ}$ angle of attack, no leading-edge vortex can be seen at any tap locations. The loss of a pressure peak on the canard upper surface is the source of the second break seen 
in the TCR pitch moments.

Figures 16 (d)-(f) show the pressure distribution over the wing surface at different chordwise locations. These figures show that as the angle of attack increases the wing vortices become larger (stronger) and the vortex cores move inboard. As vortices move downstream they become larger as well; however, the pressure coefficients become less negative. At $8^{\circ}$ angle of attack, two separate vortices can bee seen over the wing. At higher angles of attack and downstream positions, only one vortex is present because the wing vortices merge upstream.

The sideslip sweeps were simulated using the full-aircraft mesh with zero canard angle; the sideslip angle ranges from $-16^{\circ}$ to $16^{\circ}$ and corresponds to two angels of attack of $6^{\circ}$ and $20^{\circ}$. The force and moment predictions are compared with experiments in Figure 17. The results show that CFD and experiment data match well at $6^{\circ}$. They do not match everywhere at $\alpha=20^{\circ}$, particularly for the moment coefficients. Note that at $20^{\circ}$ angle of attack, the wing vortices interact and merge and the numerical and experimental results largely depend on the total number of iterations completed. Figure 17 shows that while lateral coefficients (side-force, roll and yaw moments) are nearly linear with side-slip changes at $\alpha=6^{\circ}$, they become very nonlinear at $\alpha=20^{\circ}$. The high angle-of-attack data even show opposite slope signs in the lateral coefficients.

Notice that while the computed longitudinal aerodynamic coefficients are symmetric with respect to the side-slip angle, the experimental pitch moment data are not and differ at positive and negative side-slip angles, as shown in Figure 17 (b). These differences are even larger for $\alpha=6^{\circ}$. Note that no vortex breakdown occurs at these angles; the vortices are stationary in their locations over the wing and canard surfaces as well. The authors are not sure about the cause of the non-symmetric behavior seen in the experimental pitch moments.

Figure 18 shows the flow solution at extreme sideslip conditions and $\alpha=6$ and $20^{\circ}$. Note that all vortex structures are asymmetric. At $\beta=16^{\circ}$ and $\alpha=6^{\circ}$, the fuselage vortex is shifted towards the left wing side. The canard and wing show much stronger vortices on the left side than on the right side. The vortex cores are moved to the left as well. A vortex can also be seen over the left-side of the vertical tail originating from the tail apex. At $\beta=16^{\circ}$ and $\alpha=20^{\circ}$, the right wing vortices interact and merge to a single large vortex. However, no merged vortex can be seen on the left wing.

To investigate the effects of the gap regions in the overset mesh on solutions, the CFD data from the single and overset meshes are compared for an angle-of-attack sweep. Figure 19 shows that these data are very close, though the overset mesh was only run for 2,000 iterations. Figure 20 also compares the surface solution of both meshes at 20 degrees angle of attack. Solutions of both meshes look similar; the overset mesh predicts a slightly larger canard vortex than the single mesh.

The overset mesh was then used to investigate and validate CFD data at different canard angles. These angles include $\phi_{c}=\left[-30^{\circ},-10^{\circ}, 0^{\circ}, 10^{\circ}\right]$. The normal force and pitch moment coefficients were compared with available experimental data in Figure 21. The results show that predictions from CFD using an overset grid method match very well with experiments. Note that the normal force and pitch moment increase with increasing canard angle. The 
curve slopes are similar for small angles of attack, but they become nonlinear with respect to canard angle at higher angles of attack. The canard effects on the TCR flowfield can be seen in Figure 22 at 20 degrees angle of attack. As the canard angle decreases, the canard vortex becomes smaller and therefore the fuselage and LE vortex become larger. Notice that at $\phi_{c}=-30^{\circ}$, no vortex is seen over the canard.

\subsection{Dynamic Results}

Final results correspond to validation of CFD data for forced-harmonic pitching motions. These motions are large amplitude motions defined as: $\alpha=8^{\circ}+10^{\circ} \sin (\omega t)$ and $\alpha=8^{\circ}+$ $20^{\circ} \sin (\omega t)$ with frequencies of $0.5,1.0$, and $1.5 \mathrm{~Hz}$. These simulations are second order accurate in time with a Newton sub-iteration scheme to improve time accuracy of the point implicit method and approximate Jacobians. In this work, dynamic motion runs were made with five Newton sub-iterations. All dynamic simulations were performed for two cycles with a non-dimensional time-step of $\Delta t^{*}=0.01$, where $t^{*}=\frac{V t}{c}$. For more details of time step selection, the reader is referred to the work of Cummings et al.[32].

CFD solutions are compared with experiments in Figs. 23 and 24. The plots show that hysteresis loops are formed in the normal force and pitch moment curves. The normal force loop is clockwise but the pitch moment loop is traversed in a counter clockwise direction. The plots also show that the hysteresis loops have nonlinear shape and they become larger as the frequency increases. Even for these unsteady motions in a nonlinear angle-of-attack regime, reasonable agreement was found between CFD and experiments with some exceptions at large angles of attack.

Figure 25 shows in more detail a few TCR flow solutions during the upstroke and the downstroke motion of $\alpha=8^{\circ}+20^{\circ} \sin (\omega t)$ with $f=1.5 \mathrm{~Hz}$ frequency. It can be seen that the wing has larger vortex during the upstroke (points B and D in the plot) compared with the downstroke (points $\mathrm{A}$ and $\mathrm{C}$ in the plot) motion at about the same angle of attack. During the upstroke, the wing has higher angles of attack due to pitch rate effects (local vertical velocity component will vary with pitch rate and distance from pitching point). And for the same reason the canard should have a smaller vortex during the upstroke compared with the downstroke motion at about the same angle of attack. However, this is not the case for angles about $\alpha=20^{\circ}$ (points $\mathrm{A}$ and B in Figure 25). At this and higher angles, the canard vortex is away from the surface and has no effect on the canard pressure distribution. For angles slightly less than $\alpha=20^{\circ}$, no canard vortex can still be seen during the downstroke. This is mainly due to time-delay effects during pitching motions.

\section{Conclusions}

This work presented the CFD study of a canard-configured TransonicCruiser. The low speed aerodynamic characteristics of the TCR are available from the SimSAC project and were used in this work to validate CFD predictions. All tests were run at a free-stream velocity of $40 \mathrm{~m} / \mathrm{s}$, which corresponds to a sea level Mach number of 0.117 , and a Reynolds number of 0.778 million based on the mean aerodynamic chord of the wind tunnel model. 
The TCR wind tunnel experiments only include total integrated forces/moments; the surface pressure measurements and flowfield information are not available from these tests.

An overset grid method was used to simulate canard deflections available in the experiments, however, a gap region is required between the canard (overset mesh) and the aircraft (background mesh) to allow the mesh assembly. The CFD results show that the gap does not significantly affect the aerodynamic predictions. Numerical data with and without the canard were compared with static and dynamic experimental data. Overall, a good agreement was found between CFD and experiments for most cases. The CFD data showed a dip in longitudinal coefficients between $\alpha=18^{\circ}$ and $\alpha=24^{\circ}$, that is not present in the experimental data. This dip becomes smaller for a canard-configured TCR. The occurrence of these dips depends on the number of time steps or iterations completed; dips could not be seen for short run simulations. Other discrepancies between CFD and experiment were seen in the sideslip angle sweeps at $\alpha=20^{\circ}$.

Flowfield solutions showed that vortices are formed over the wing, LEX, and fuselage of the canardless TCR at moderate to high angles of attack. The wing vortex starting point becomes fixed at the wing apex; it moves toward the LEX vortex as the angle of attack increases. At about $\alpha=20^{\circ}$, the wing and LEX vortices interact and merge. For angles between $\alpha=18^{\circ}$ and $\alpha=24^{\circ}$, the merging point changes and then is nearly fixed at higher angles. The dip seen in the coefficients was related to the wing vortices interaction and merging. At high angles of attack, the fuselage vortex interacts with the wing vortices as well. For a canard-configured TCR, a vortex is formed over the canard that changes the wing/fuselage vortices and aerodynamic performance. The canard downwash causes smaller LEX and fuselage vortices. At high angles of attack, however, the canard vortex produces stronger merged vortices which increases the maximum lift and delays the vortex breakdown.

The dynamic results showed that hysteresis loops were formed in the normal force and pitch moment curves. The normal force loop was clockwise but the pitch moment loop was traversed in a counter clockwise direction. The plots also showed that the hysteresis loops had nonlinear shape due to vortex formations and pitch rate and time-delay effects. Reasonably good agreement between CFD and experiments is obtained for the dynamic cases.

\section{Acknowledgements}

Mehdi Ghoreyshi is supported by USAFA under contract FA70001320018; their financial support is gratefully acknowledged. Acknowledgements are expressed to the Department of Defense High Performance Computing Modernization Program (HPCMP), ERDC for providing computer time. The authors appreciate the support provided by the High Performance Computing Research Center at USAFA. Special thanks are also due to Art Rizzi of KTH, Jan Vos of CFSE Engineering and Sergio Ricci of Polimi for providing the TCR CAD and experimental data. 


\section{References}

[1] Rizzi, A., Eliasson, P., Goetzendorf-Grabowski, T., Vos, J. B., Zhang, M., and Richardson, T. S., "Design of a Canard Configured TransCruiser Using CEASIOM," Progress in Aerospace Sciences, Vol. 47, No. 8, 2011, pp. 695-705 DOI :10.1016/j.paerosci.2011.08.011.

[2] Mani, M., Rider, B. J., Sclafani, A. J., Winkler, C., Vassberg, J., Dorgan, A. J., Cary, A., and Tinoco, E. N., "Reynolds-Averaged Navier Stokes Technology for Transonic Drag Prediction: A Boeing Perspective," Journal of Aircraft, Vol. 51, No. 4, 2014, pp. 1118-1134 DOI : $10.2514 / 1 . C 032717$.

[3] Rizzi, A., P., E., McFarlane, C., Goetzendorf-Grabowski, T., and Vos, J., "Virtual-Aircraft Design \& Control of TransCruiser a Canard Configuration," AIAA Paper 2010-8245, August 2010.

[4] Von Kaenel, R., Rizzi, A., Oppelstrup, J., Grabowski, T., Ghoreyshi, M., Cavagna, L., and Bérard, A., "CEASIOM: Simulating Stability \& Control with CFD/CSM in Aircraft Conceptual Design," 26th International Congress of the Aeronautical Sciences, ICAS, 14 - 19 September 2008, Anchorage, Alaska, USA.

[5] Eliasson, P., DaRonch, A., Vos, J. B., Zhang, M., and Rizzi, A., "Virtual Aircraft Design of TransCruiser Computing Break Points in Pitch Moment Curve," AIAA Paper 2010-4366, June-July 2010.

[6] Da Ronch, A., Vallespin, D., Ghoreyshi, M., and Badcock, K. J., "Evaluation of Dynamic Derivatives Using Computational Fluid Dynamics," AIAA Journal, Vol. 50, No. 2, 2012, pp. 470484 DOI : $10.2514 / 1 . J 051304$.

[7] Samimi, S., Davari, A. R., and Soltani, M. R., "Canard-Wing Interactions in Subsonic Flow," IJST, Transactions of Mechanical Engineering, Vol. 37, No. 2, 2013, pp. 133-147.

[8] Khrabrov, A., Kolinko, K., Zhuk, A., and Grishin, I., "Wind Tunnel Test Report," SimSAC Technical Report, October 2009.

[9] Torenbeek, E., Synthesis of Subsonic Airplane Design, Delft University Press, Netherlands, 1982.

[10] Gloss, B. B. and McKmney, L. W., "Canard Wing Lift Intereference Related to Maneuvering Aircraft At Subsonic Speeds," NASA Technical Report, NASA TM X-2897, December 1973.

[11] Phillips, W. F., Mechanics of Flight, Aerospace/Engineering, Wiley, 2004.

[12] Zhang, G. Q., Yu, S. C. M., Chien, A., and Yang, S. X., "Aerodynamic Characteristics of CanardForward Swept Wing Aircraft Configurations," Journal of Aircraft, Vol. 50, No. 2, 2013, pp. 378-387 DOI :10.2514/1.C031740.

[13] Fleeman, E. L., Tactical Missile Design, AIAA education series, American Institute of Aeronautics and Astronautics, 2006.

[14] Roskam, J., "Airplane Design," 1990, Roskam Aviation and Engineering Corporation, Kansas, USA.

[15] Tu, E. L., "Effect of Canard Deflection on Closed-Coupled Canard-Wing-Body Aerodynamics," Journal of Aircraft, Vol. 31, No. 1, 1994, pp. 138-145 DOI : $10.2514 / 3.46466$.

[16] O'leary, J. F., Flowfield Study of a Close-Coupled Canard Configuration, Master's thesis, Naval Postgraduate School, June 1992.

[17] Pamadi, B. N., Performance, Stability, Dynamics, and Control of Airplanes, AIAA Education Series, AIAA, Reston, Virginia, 2004.

[18] Rom, J., High Angle of Attack Aerodynamics: Subsonic, Transonic, and Supersonic Flows, Springer New York, 2012.

[19] Bergamnn, A. and Hummel, D., "Aeodynamic Effects of Cnard Position On a Wing Body Configuration In Symmetrical Flow," AIAA Paper 2001-0116, January 2001.

[20] Strang, W. Z., Tomaro, R. F., and Grismer, M. J., "The Defining Methods of Cobalt: A Parallel, Implicit, Unstructured Euler/Navier Stokes Flow Solver," AIAA Paper 1999-0786, 1999.

[21] Gottlieb, J. J. and Groth, C. P. T., "Assessment of Riemann Solvers For Unsteady One-dimensional Inviscid Flows of Perfect Gasses," Journal of Fluids and Structure, Vol. 78, No. 2, 1998, pp. 437-458 DOI :10.1016/0021-9991(88)90059-9. 
[22] Tomaro, R. F., Strang, W. Z., and Sankar, L. N., "An Implicit Algorithm For Solving Time Dependent Flows on Unstructured Grids," AIAA Paper 1997-0333, 1997.

[23] Spalart, P. R. and Allmaras, S. R., "A One Equation Turbulence Model for Aerodynamic Flows," AIAA Paper 1992-0439, January 1992.

[24] Wilcox, D. C., "Reassesment of the Scale Determining Equation for Advanced Turbulence Models," AIAA Journal, Vol. 26, No. 11, 1988, pp. 1299-1310 DOI $: 10.2514 / 3.1004$.

[25] Menter, F., "Eddy Viscosity Transport Equations and Their Relation to the $k-\varepsilon$ Model," ASME Journal of Fluids Engineering, Vol. 119, 1997, pp. 876-884 DOI: $10.1115 / 1.2819511$.

[26] Tuncer, I. H., Gulcat, U., Emerson, D. R., and Matsuno, K., Parallel Computational Fluid Dynamics 2007, Springer-Verlag,Germany, 2009.

[27] Thomas, J. L., Krist, S. T., and Anderson, W. K., "Navier Stokes Computations of Vortical Flows Over Low-Aspect-Ratio Wings," AIAA Journal, Vol. 28, No. 2, 1990, pp. 205-212 DOI :10.2514/3.10376.

[28] Gursul, I., Gordnier, R., and Visbal, M., "Unsteady Aerodynamics of Nonslender Delta Wings," Progress in Aerospace Sciences, Vol. 41, No. 7, 2005, pp. 515-557 DOI : $10.1016 /$ j.paerosci.2005.09.002.

[29] Tyssel, L., "Hybrid Grid Generation for Complex 3D Geometries," Proceedings of the 7th International Conference on Numerical Grid Generation in Computational Field Simulation, 2000, pp. 337-346.

[30] Tyssel, L., "The TRITET Grid Generation System," International Society of Grid Generation (ISGG)," Proceedings of the 10the International Conference on Numerical Grid Generation in Computational Field Simulations, 2000.

[31] Spalart, P. R. and Shur, M. L., "On the Sensitization of Turbulence Models to Rotation and Curvature," Aerospace Science and Technology, Vol. 1, 1997, pp. 297-302.

[32] Cummings, R. M., Morton, S. A., and McDaniel, D. R., "Experiences in Accurately Predicting TimeDependent Flows," Progress in Aerospace Sciences, Vol. 44, 2008, pp. 241-257. 


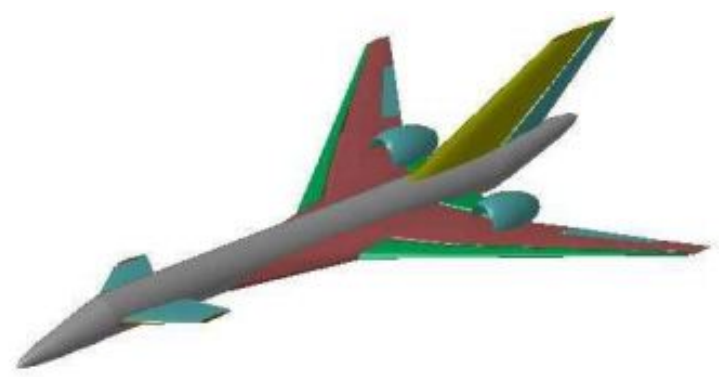

(a) TCR design concept [1]

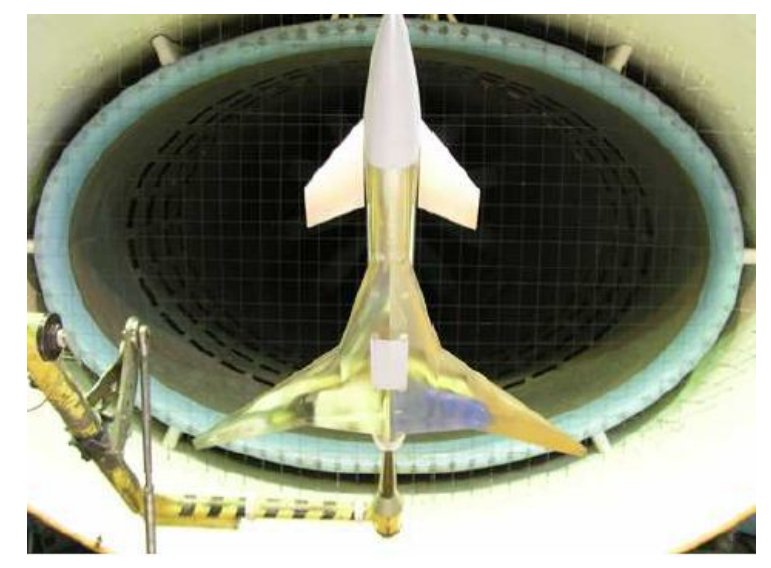

(b) TCR wind tunnel model [8]

Figure 1: TCR design and wind tunnel model. 

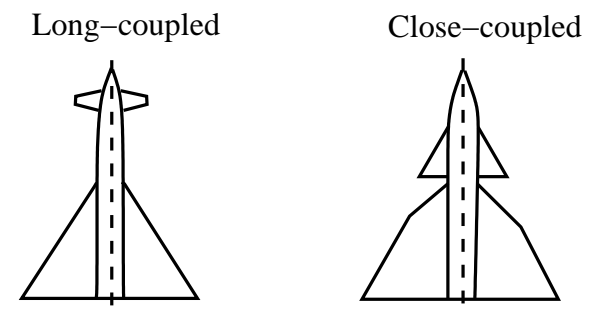

Figure 2: Canard types. Adapted from Ref. 16

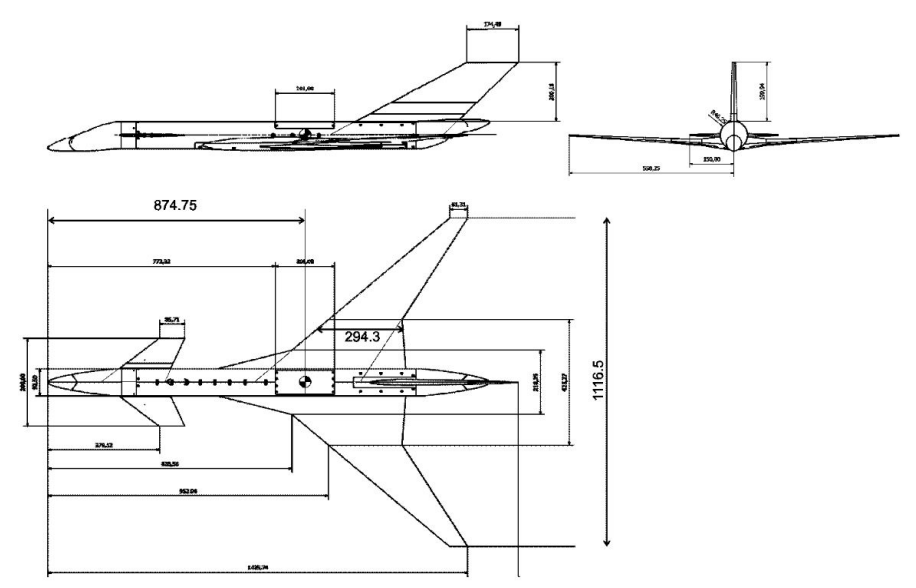

Figure 3: TCR wind tunnel model schematic. [8]

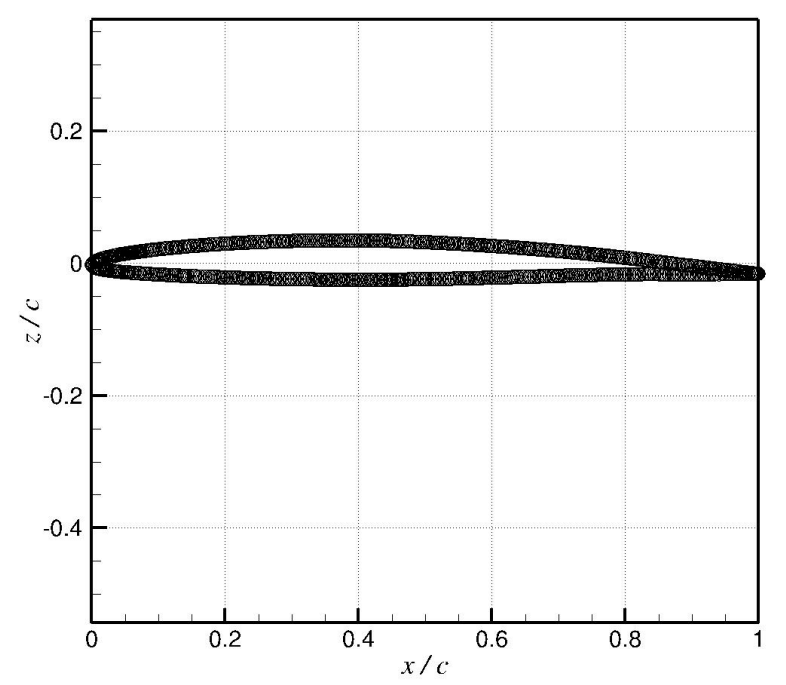

Figure 4: TCR wing airfoil section at $Y=0.3 \mathrm{~m}$. 


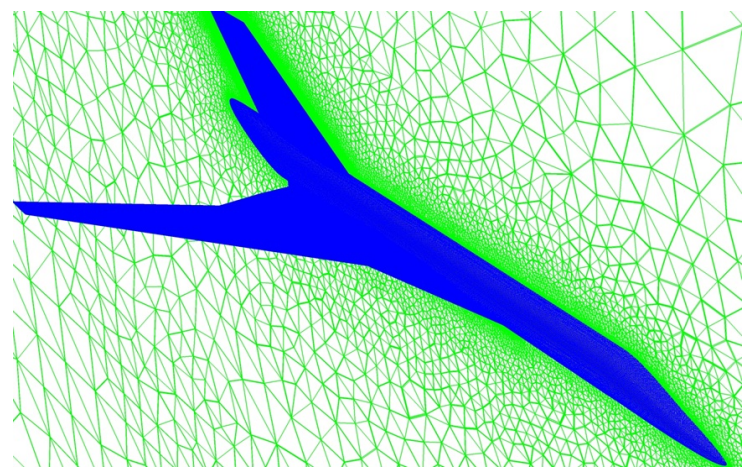

(a) without canard

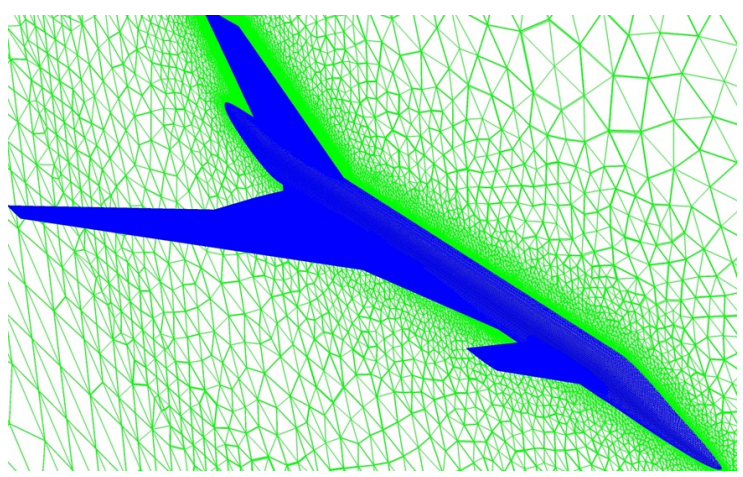

(b) with canard

Figure 5: TCR single meshes.

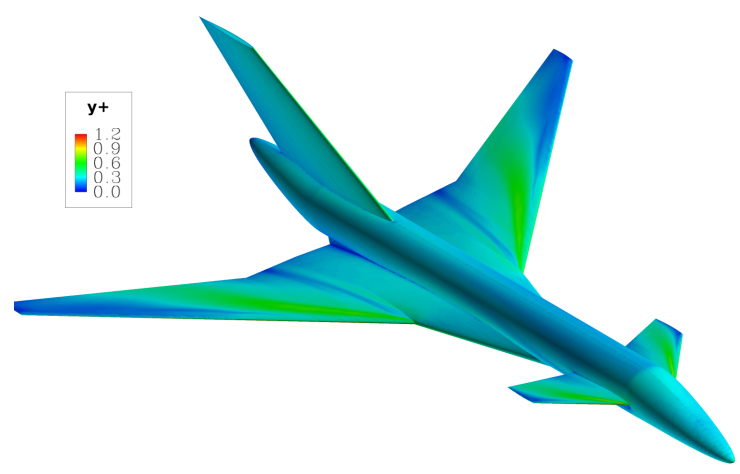

Figure 6: $y+$ of the single mesh with $\phi_{c}=0^{\circ}$ at zero degrees angle of attack.

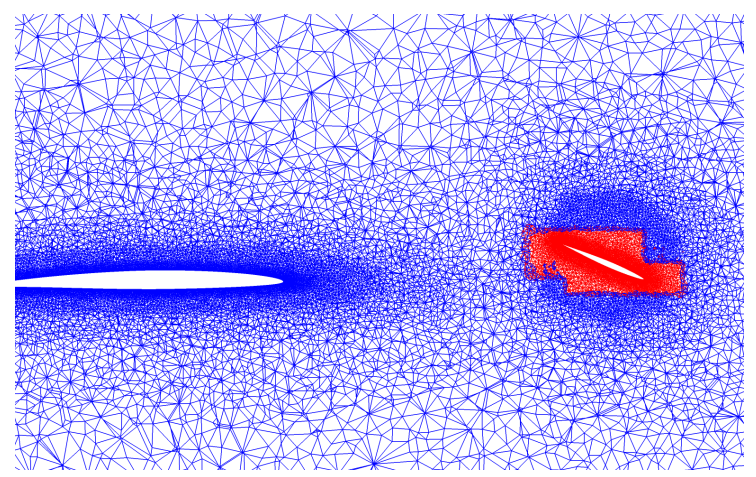

(a) canard mesh overset to the background mesh

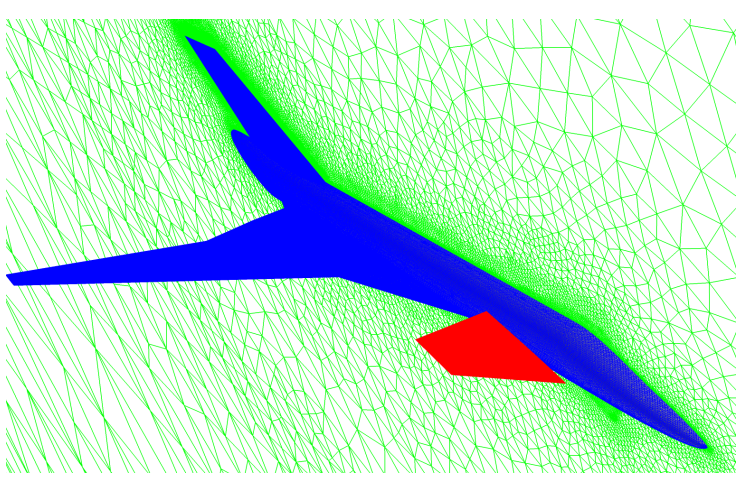

(b) $\phi_{c}=-30^{\circ}$

Figure 7: TCR overset mesh. 


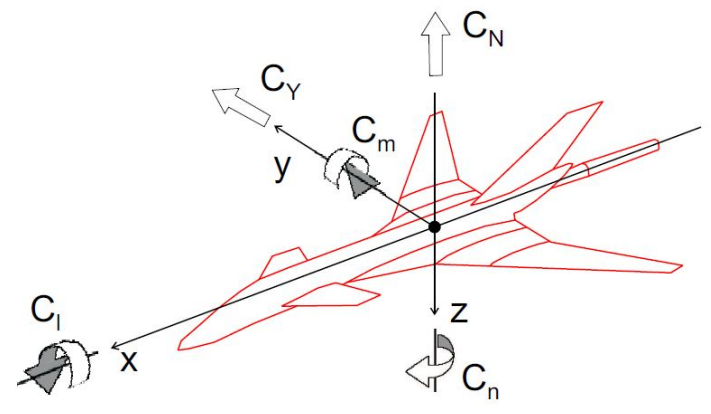

Figure 8: Body axis system for the TCR from Ref 8.

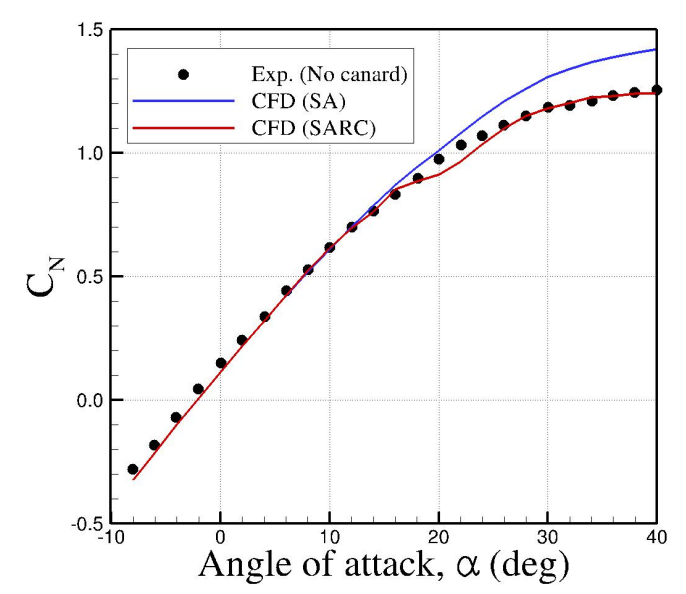

(a) normal-force coefficient

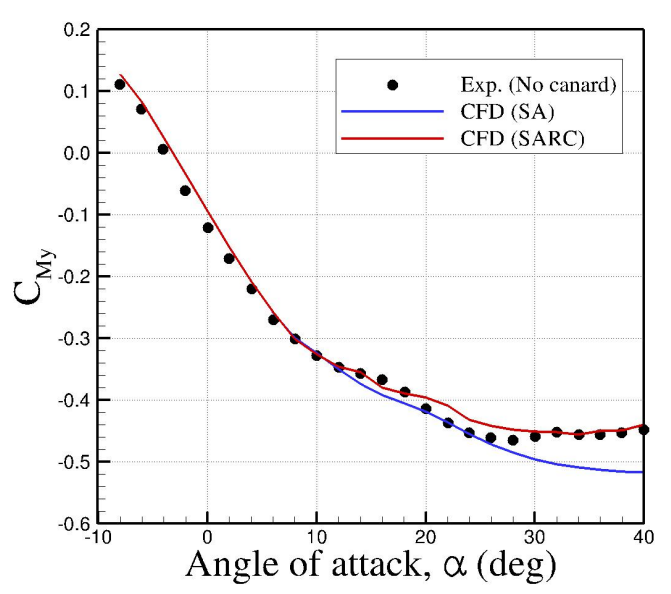

(b) pitch-moment coefficient

Figure 9: Effects of turbulence modeling on CFD prediction of the single grid without canard. 


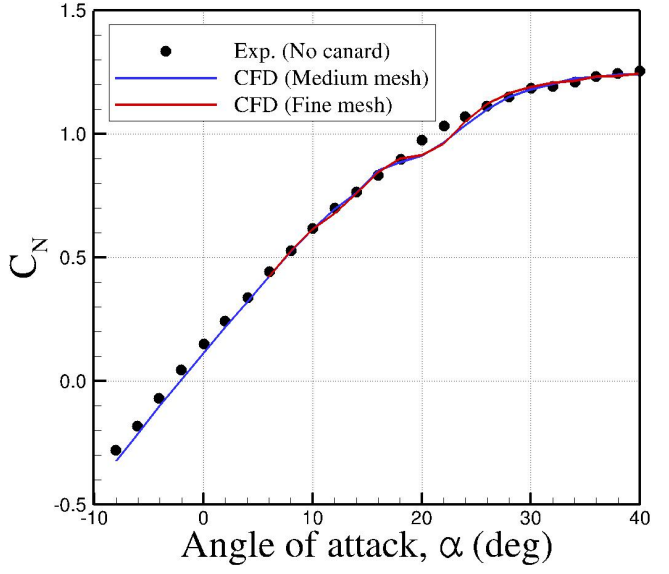

(a) normal-force coefficient

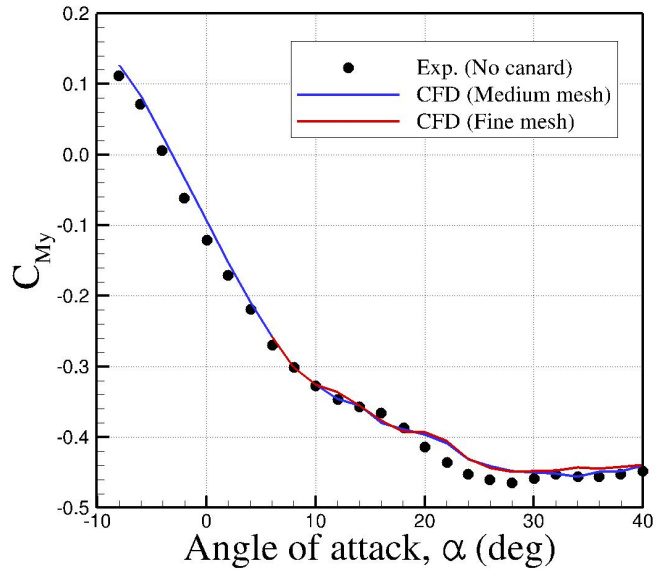

(b) pitch-moment coefficient

Figure 10: Grid convergence study for the case without canard. 


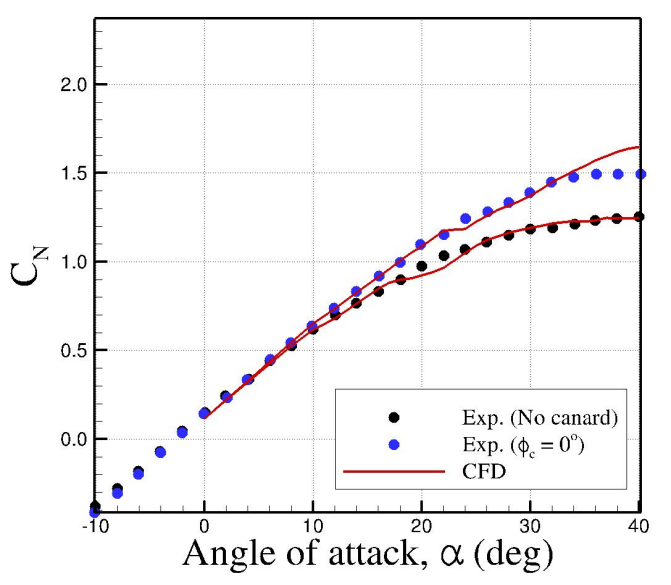

(a) normal-force coefficient

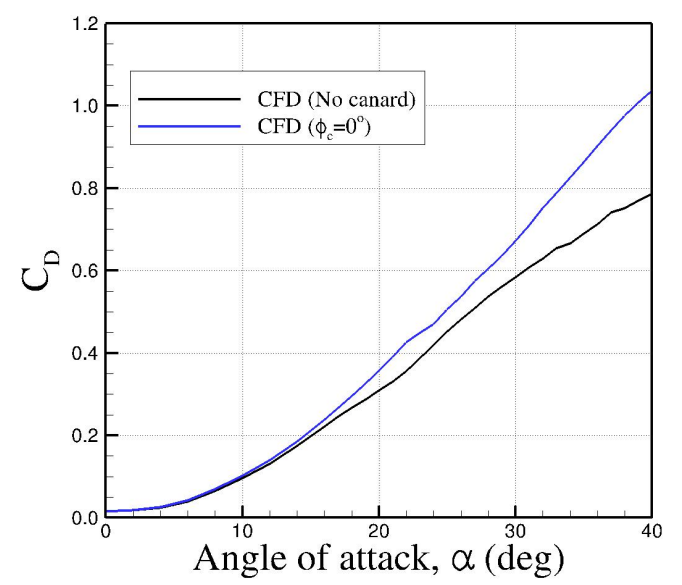

(c) Drag coefficient

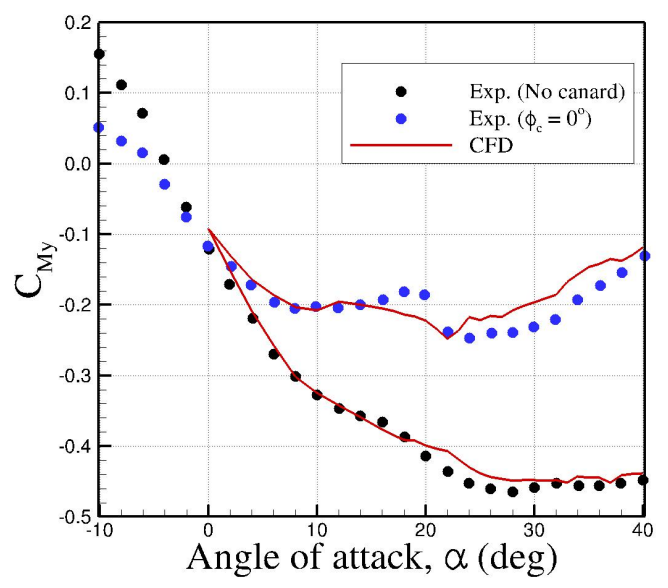

(b) pitch-moment coefficient

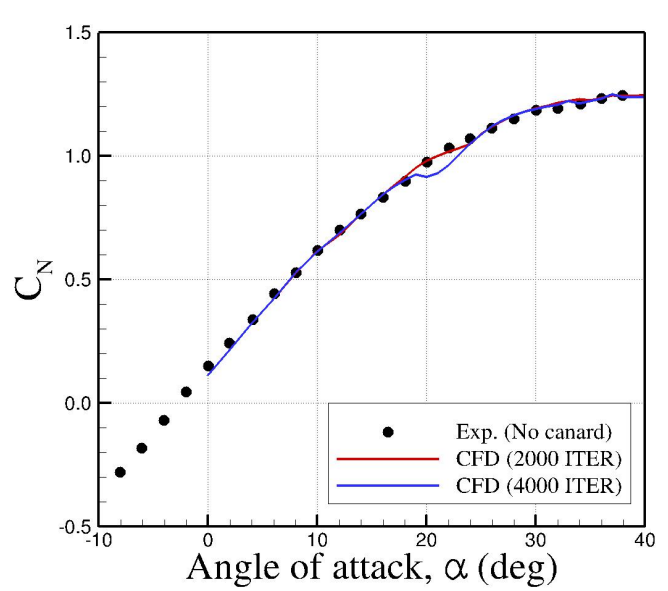

(d) Normal force changes with number of iterations

Figure 11: Sweeps of angle of attack with and without canard. All meshes are single grids. 


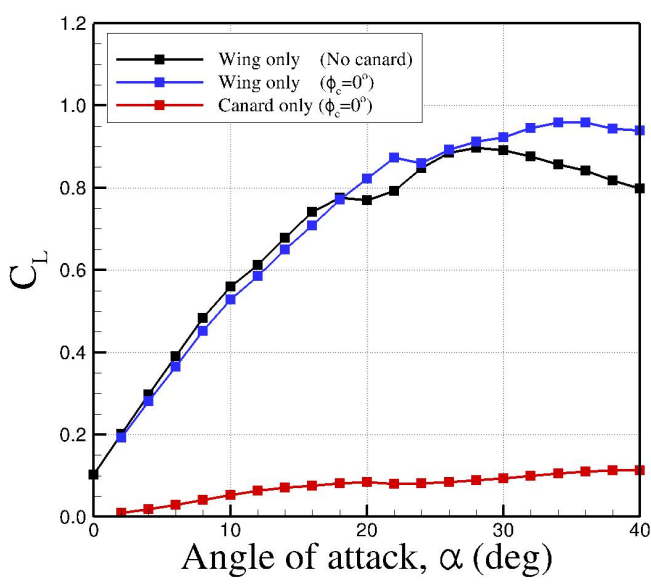

(a) normal-force coefficient

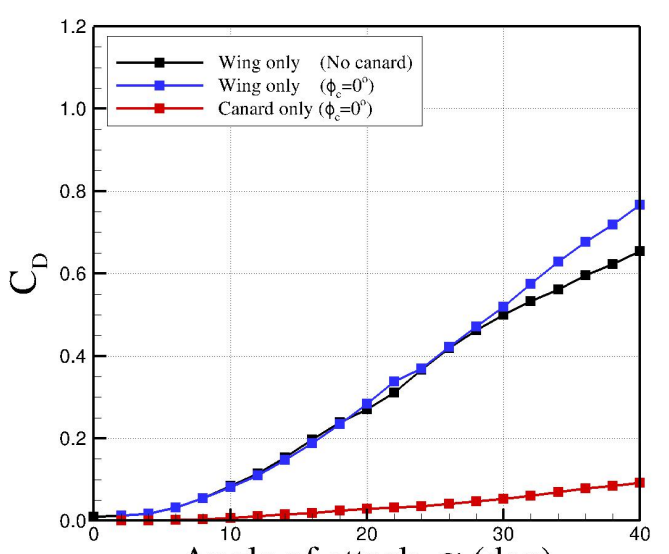

Angle of attack, $\alpha(\mathrm{deg})$

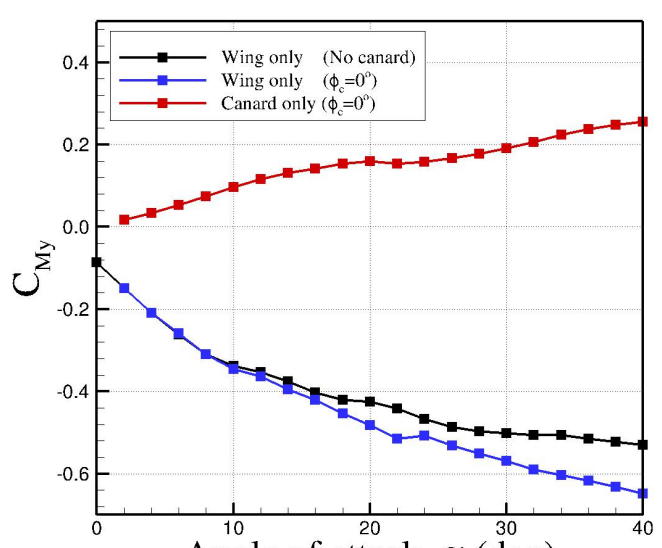

Angle of attack, $\alpha(\mathrm{deg})$

(b) pitch-moment coefficient

(c) Drag coefficient

Figure 12: Effects of canard on the wing aerodynamic performance. 


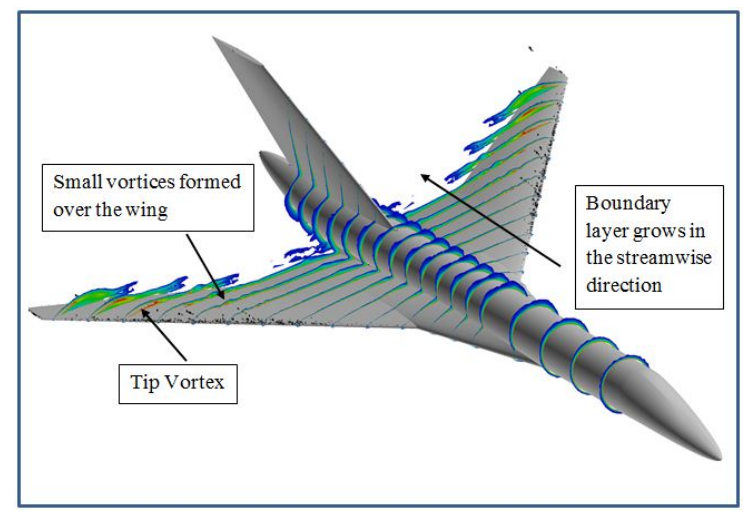

(a) $\alpha=6^{\circ}$

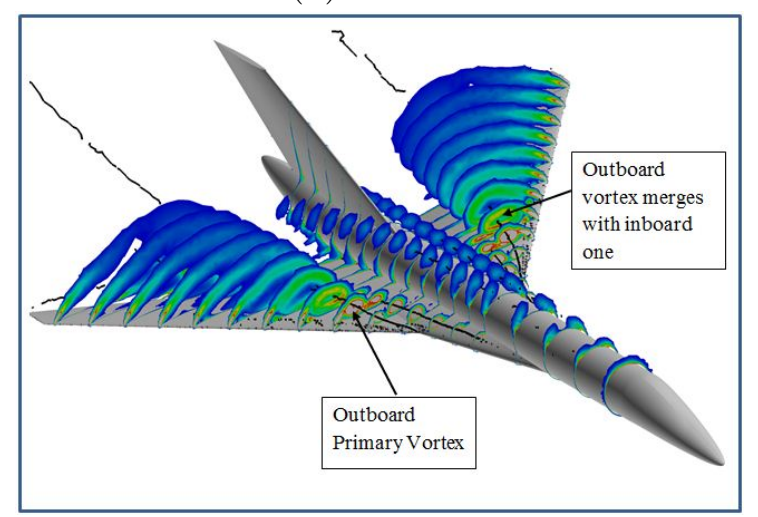

(c) $\alpha=18^{\circ}$

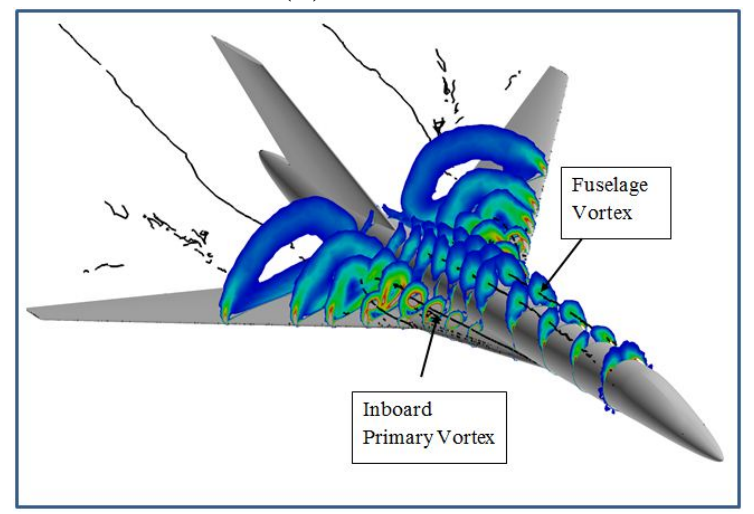

(e) $\alpha=30^{\circ}$

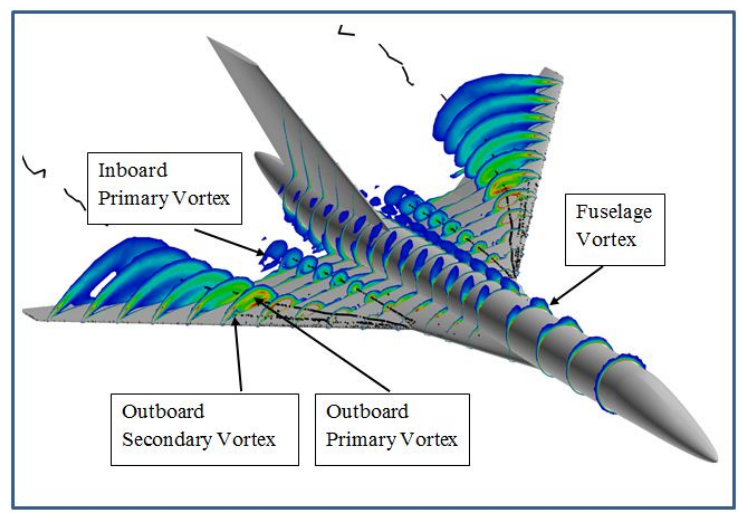

(b) $\alpha=12^{\circ}$

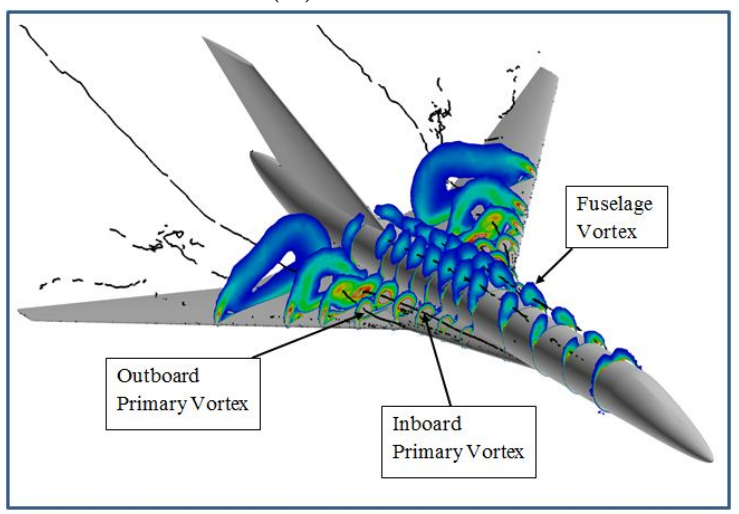

(d) $\alpha=24^{\circ}$

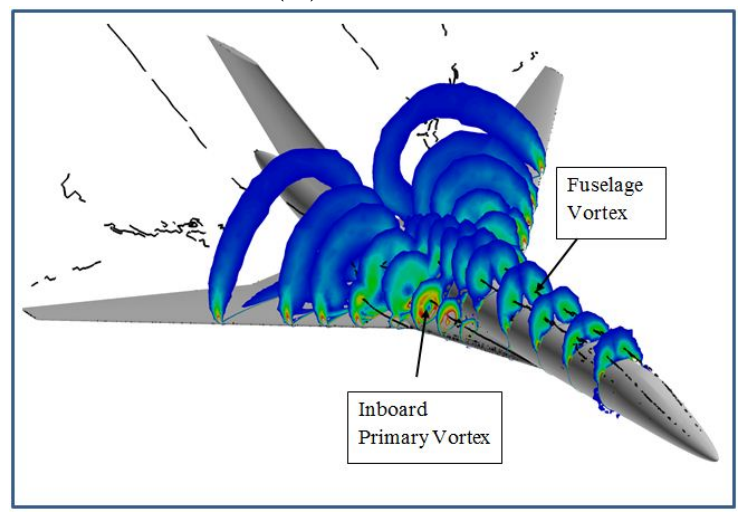

(f) $\alpha=40^{\circ}$

Figure 13: TCR (without canard) flow solutions. Iso-surfaces were created and colored by vorticity. The black lines show the core of vortices. 


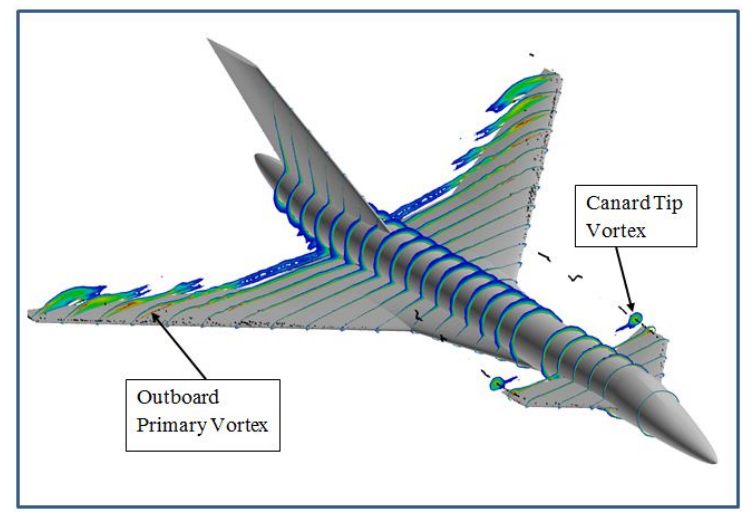

(a) $\alpha=6^{\circ}$

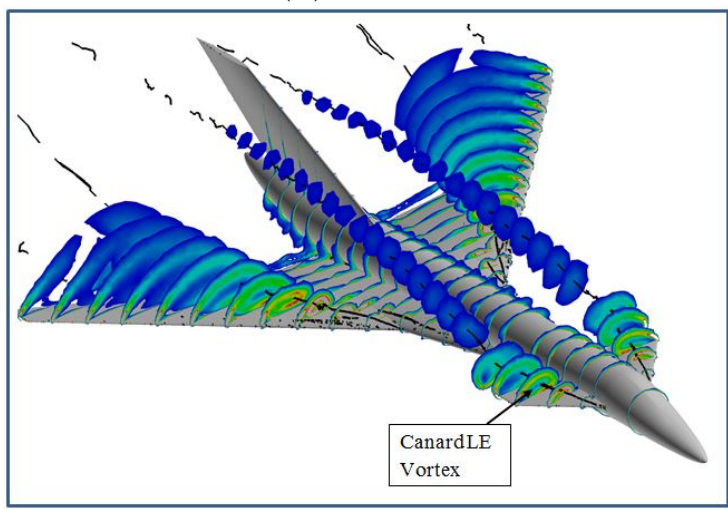

(c) $\alpha=18^{\circ}$

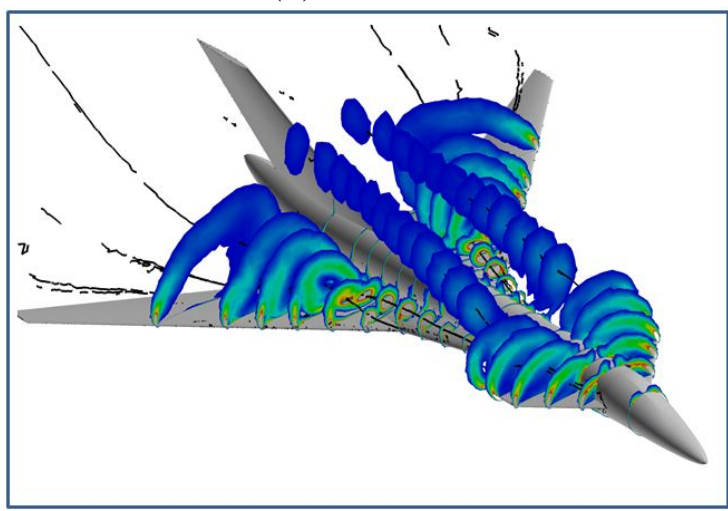

(e) $\alpha=30^{\circ}$

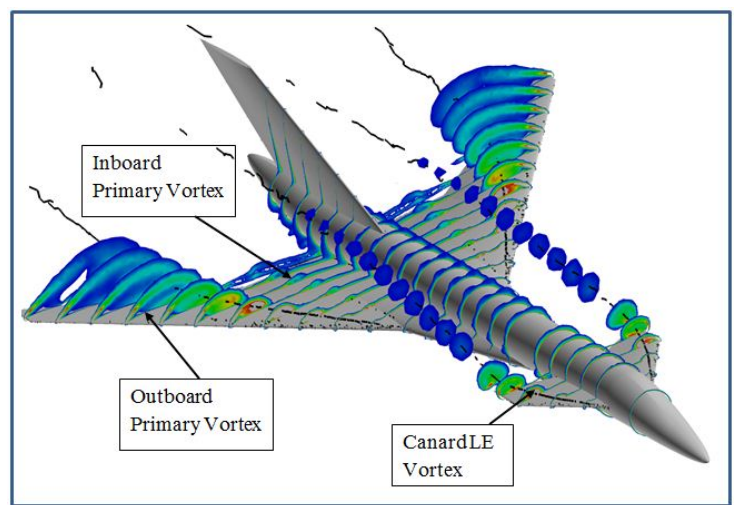

(b) $\alpha=12^{\circ}$

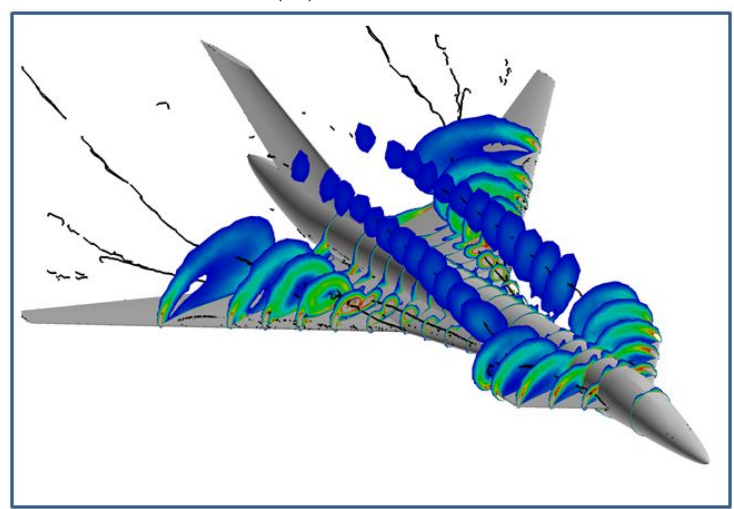

(d) $\alpha=24^{\circ}$

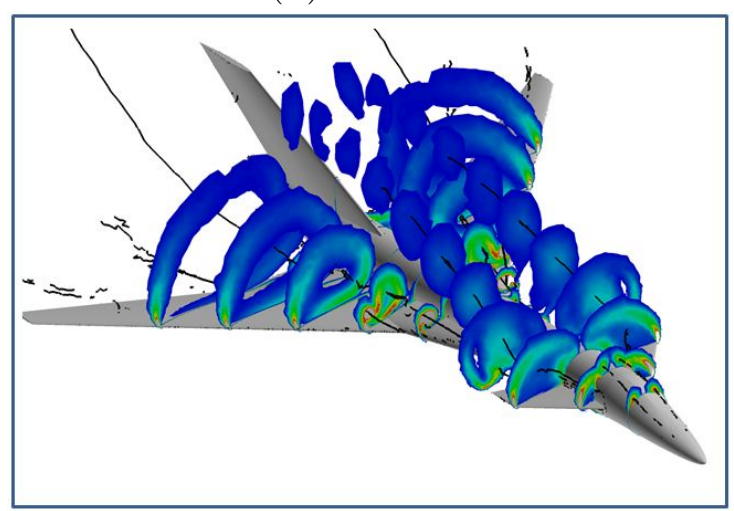

(f) $\alpha=40^{\circ}$

Figure 14: TCR $\left(\phi_{c}=0^{\circ}\right)$ flow solutions. Iso-surfaces were created and colored by vorticity. The black lines show the core of vortices. 


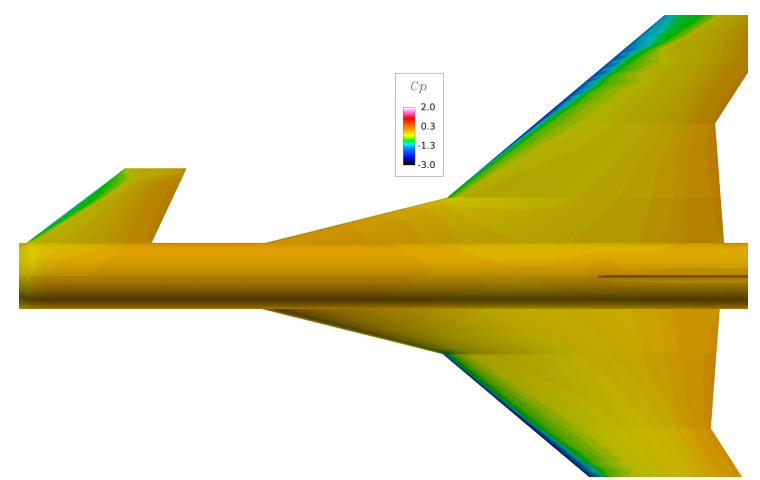

(a) $\alpha=6^{\circ}$

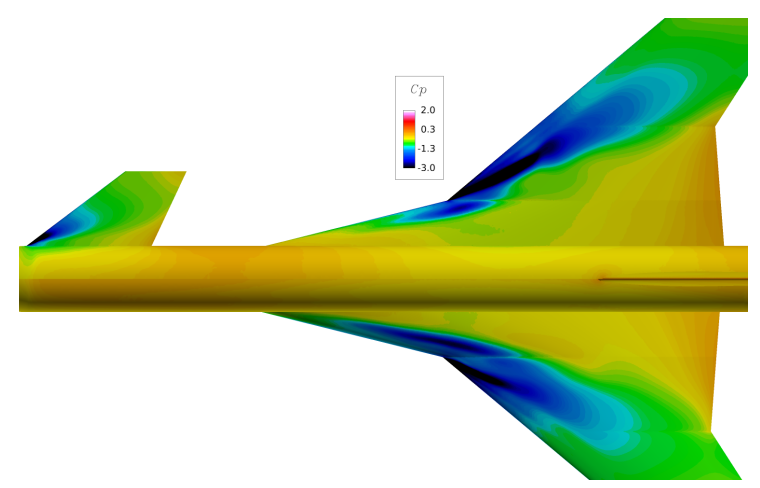

(c) $\alpha=18^{\circ}$

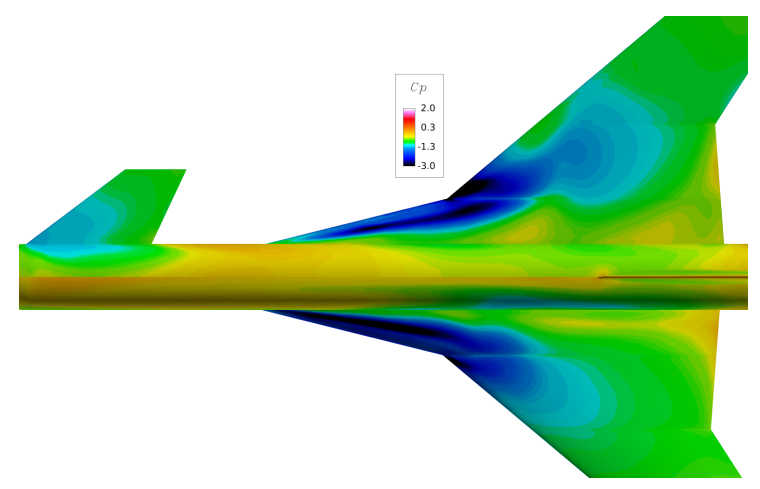

(e) $\alpha=30^{\circ}$

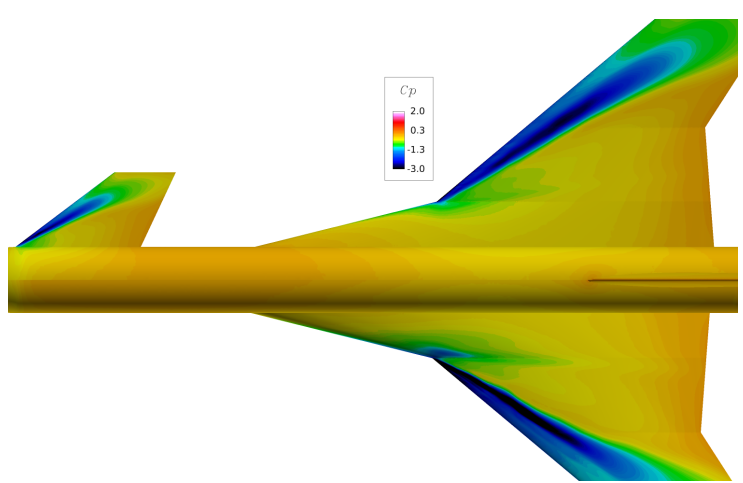

(b) $\alpha=12^{\circ}$

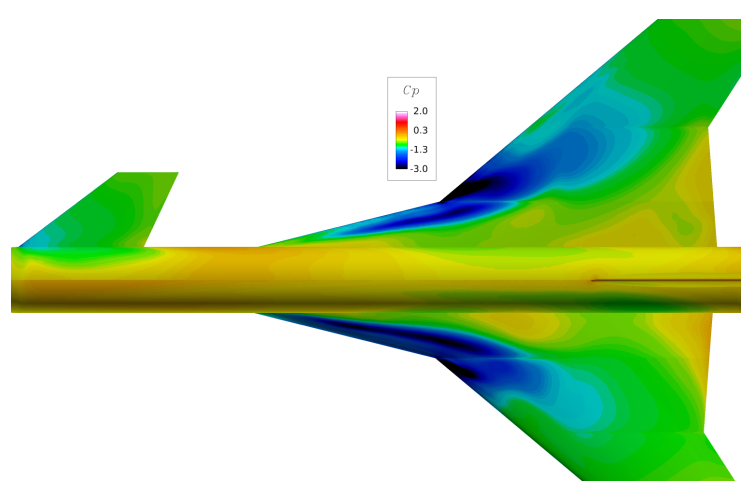

(d) $\alpha=24^{\circ}$

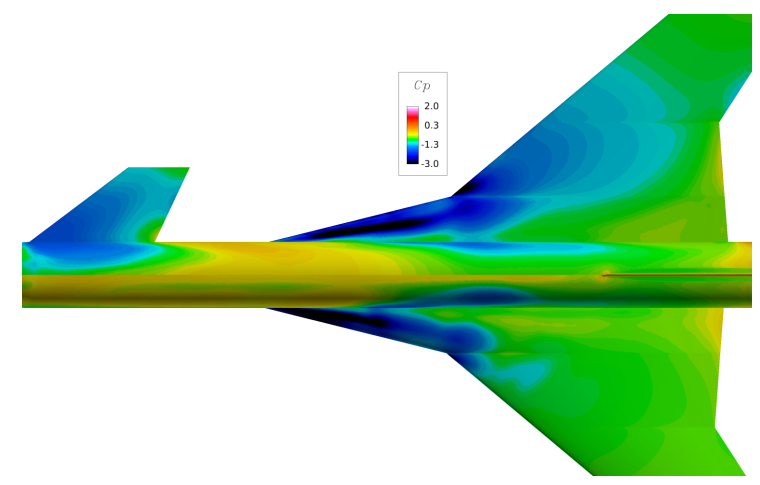

(f) $\alpha=40^{\circ}$

Figure 15: Canard on/off pressure solutions. 


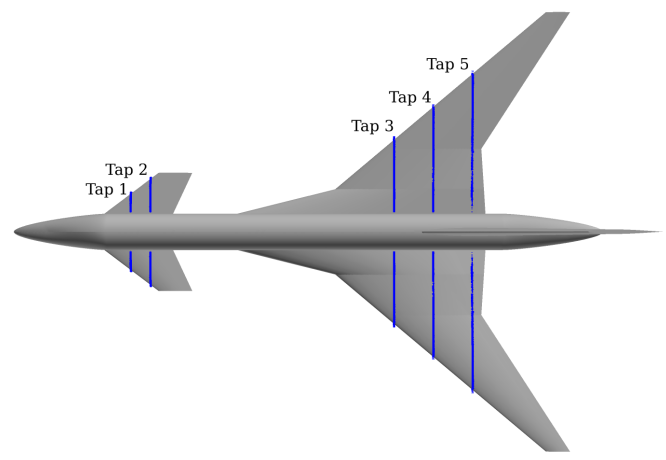

(a) Tap locations

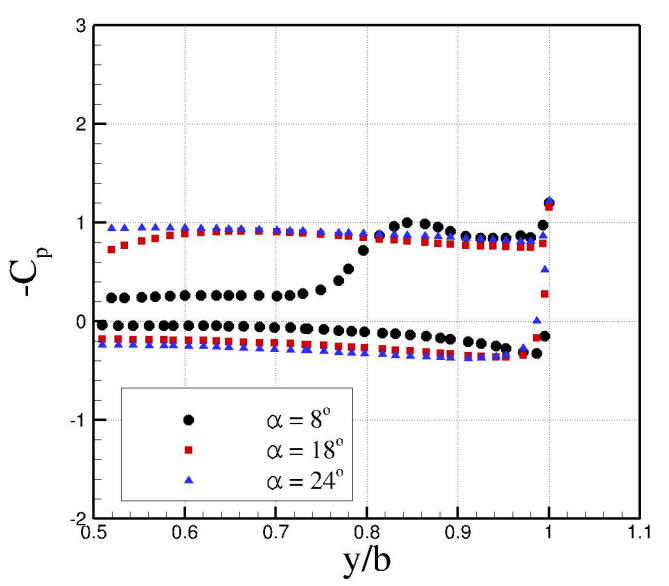

(c) Tap 2

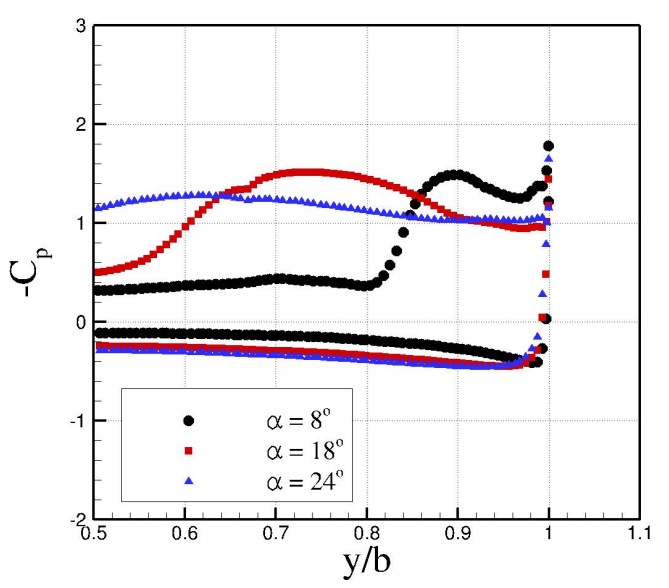

(e) Tap 4

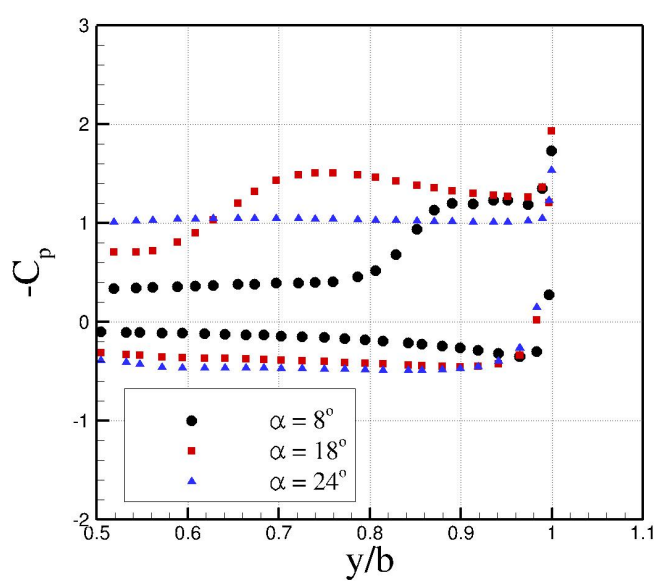

(b) Tap 1

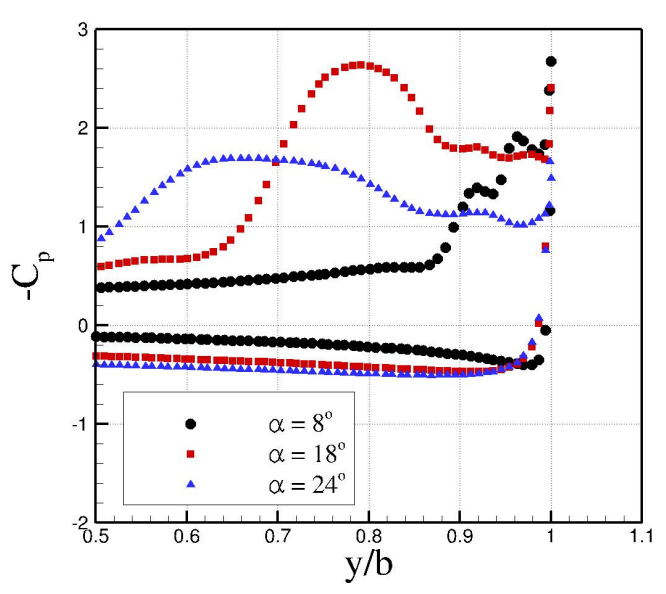

(d) Tap 3

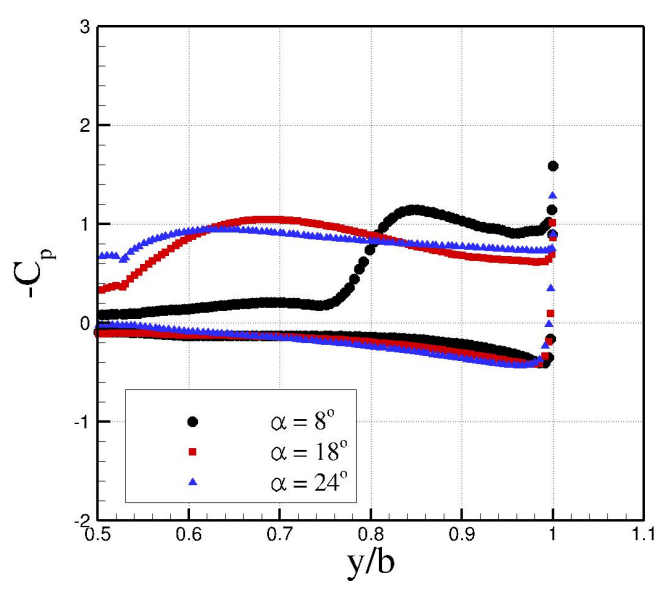

(f) Tap 5

Figure 16: Pressure coefficients of canard on 28 onfiguration at different spanwise locations. 


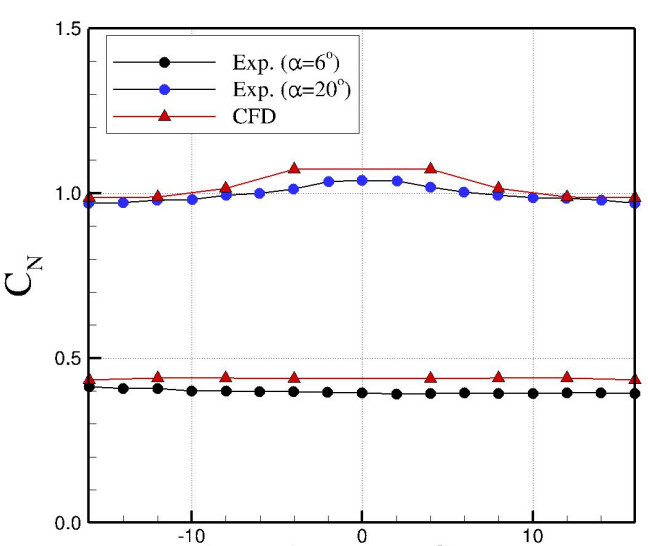

Side slip angle, $\beta$ (deg)

(a) normal-force coefficient

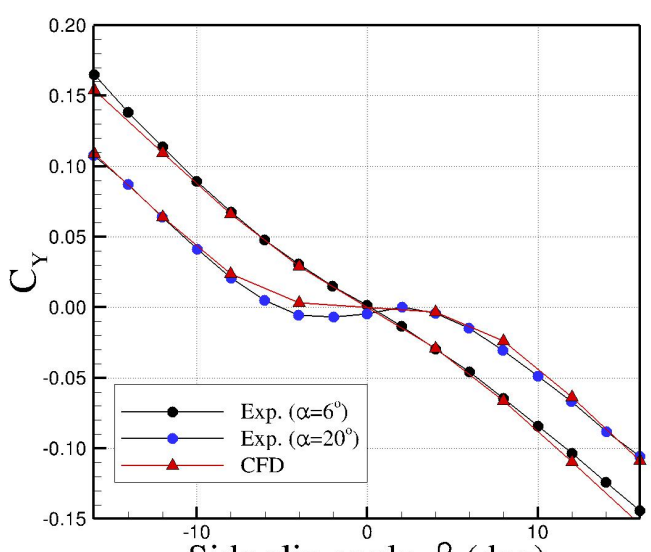

Side slip angle, $\beta$ (deg)

(c) side-force coefficient

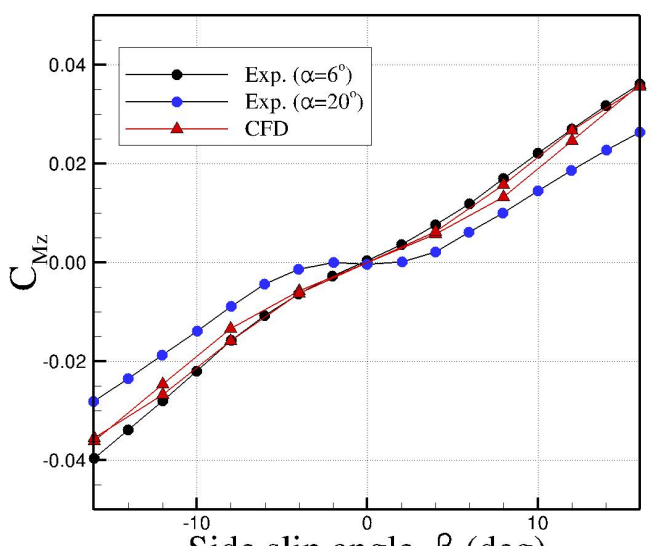

Side slip angle, $\beta$ (deg)

(e) Yaw-moment coefficient

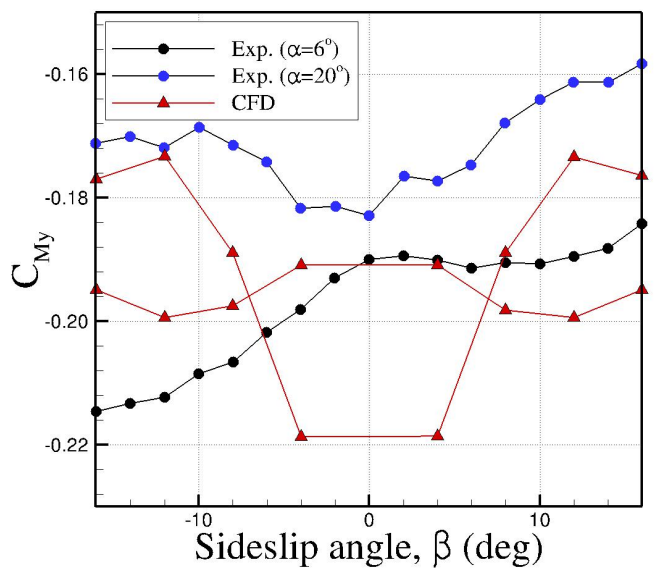

(b) pitch-moment coefficient

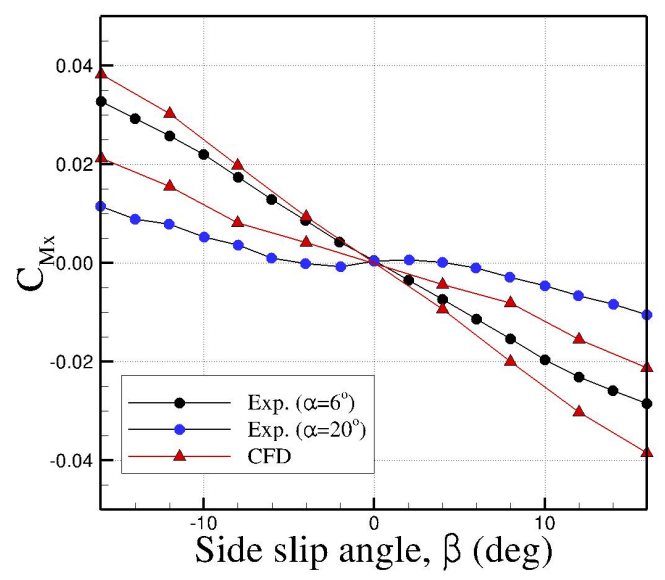

(d) roll-moment coefficient

Figure 17: Sweeps of side-slip for ${ }^{29} \alpha=6^{\circ}$ and $\alpha=20^{\circ}$ and $\phi_{c}=0^{\circ}$ 


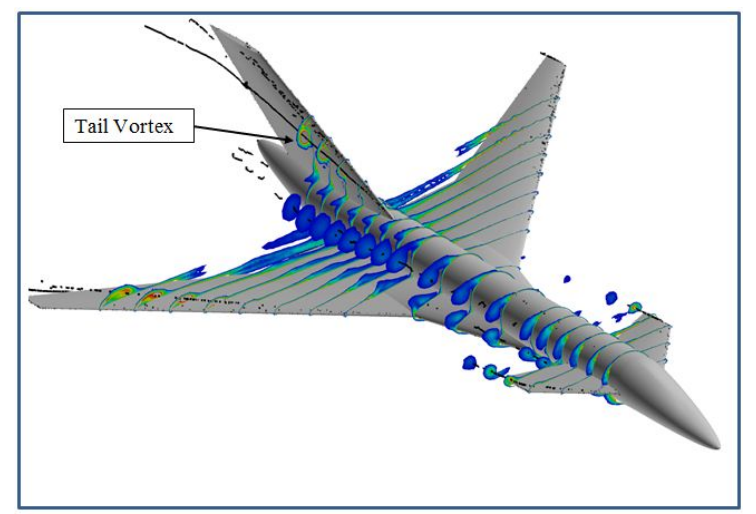

(a) $\alpha=6^{\circ}$ and $\beta=-16^{\circ}$

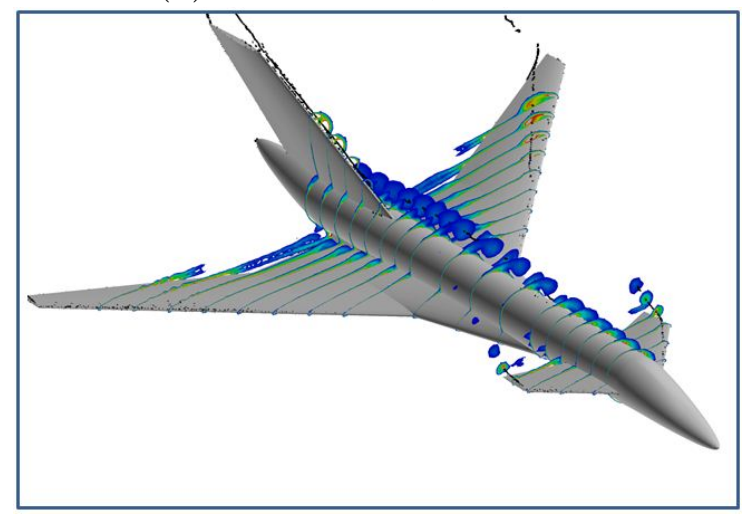

(c) $\alpha=6^{\circ}$ and $\beta=16^{\circ}$

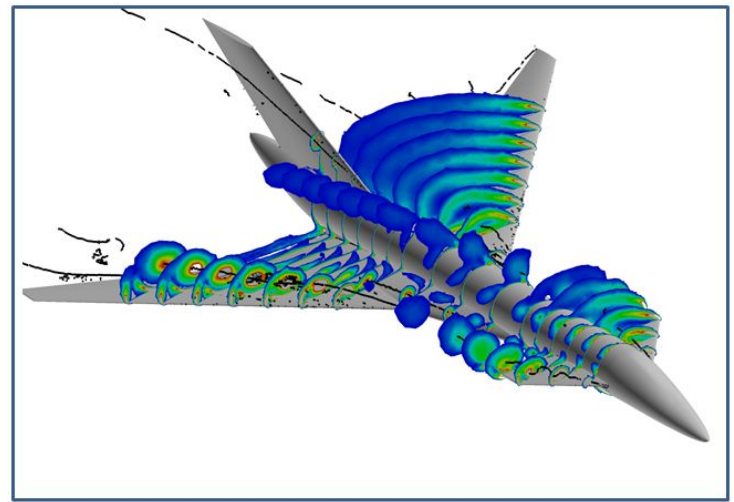

(b) $\alpha=20^{\circ}$ and $\beta=-16^{\circ}$

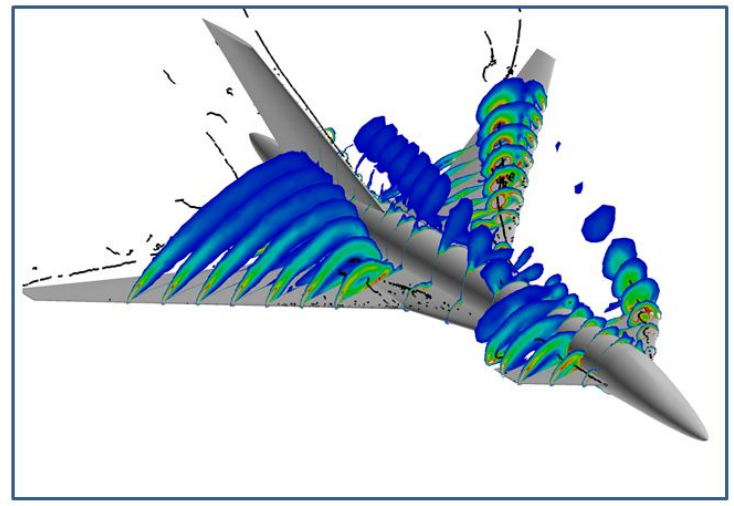

(d) $\alpha=20^{\circ}$ and $\beta=16^{\circ}$

Figure 18: TCR $\left(\phi_{c}=0^{\circ}\right)$ flow solutions at sideslip angles. Iso-surfaces were created and colored by vorticity. The black lines show the core of vortices. 


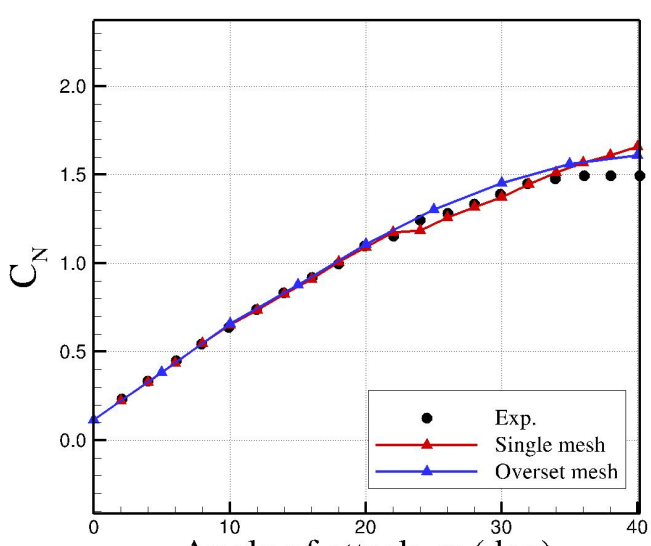

Angle of attack, $\alpha$ (deg)

(a) normal-force coefficient

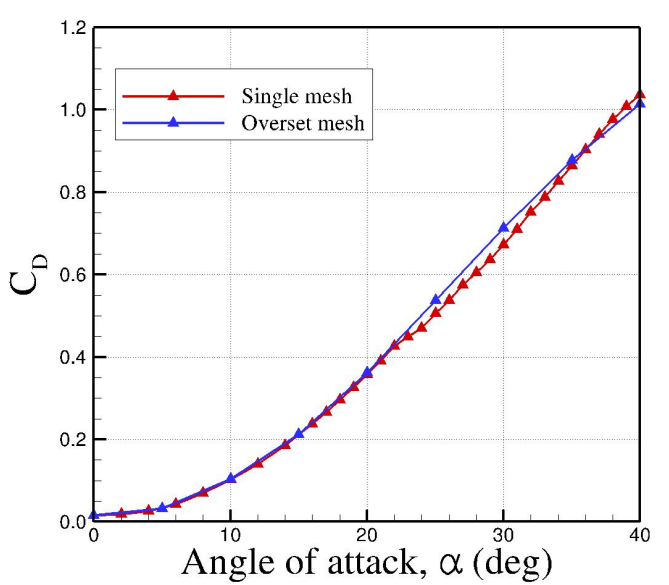

(c) Drag coefficient

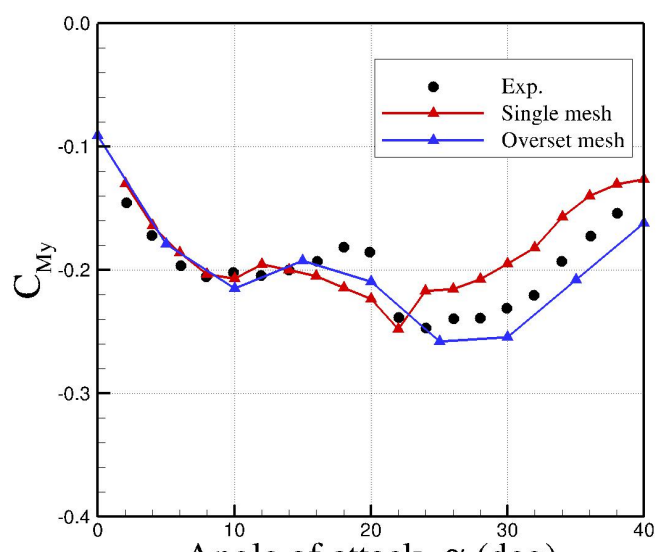

Angle of attack, $\alpha$ (deg)

(b) pitch-moment coefficient

Figure 19: Comparison of single and overset mesh prediction at $\phi_{c}=0^{\circ}$. 


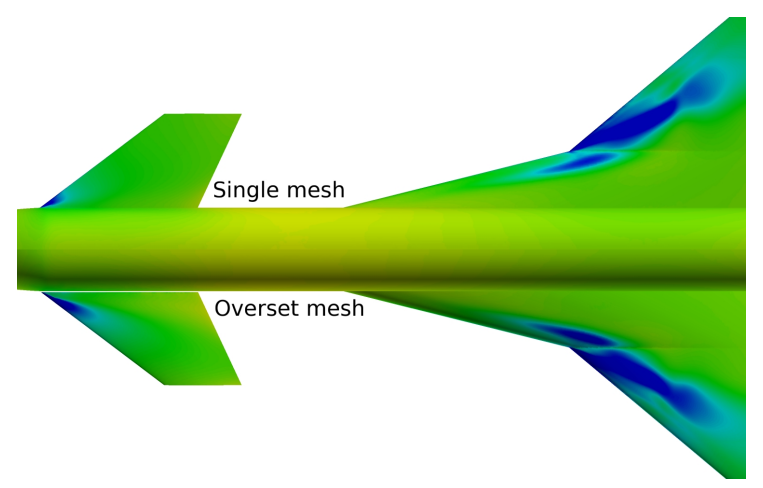

Figure 20: Effects of the canard gap on the overset flow predictions. $\alpha=20^{\circ}$ and $\phi_{c}=0^{\circ}$.

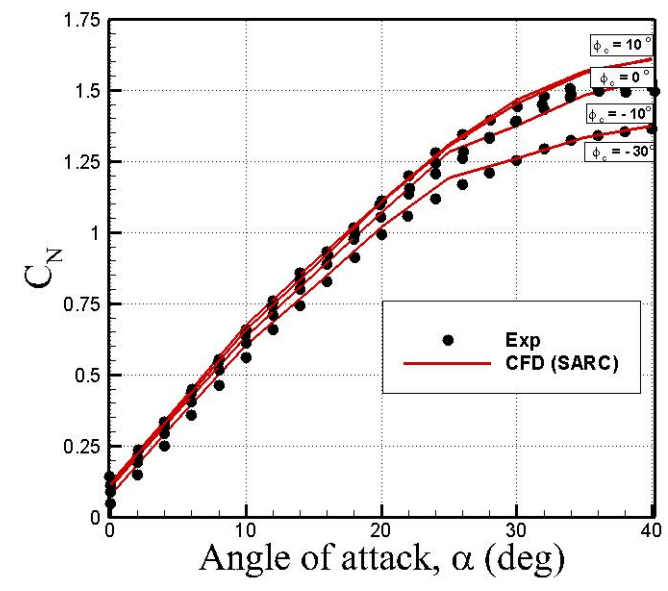

(a) normal-force coefficient

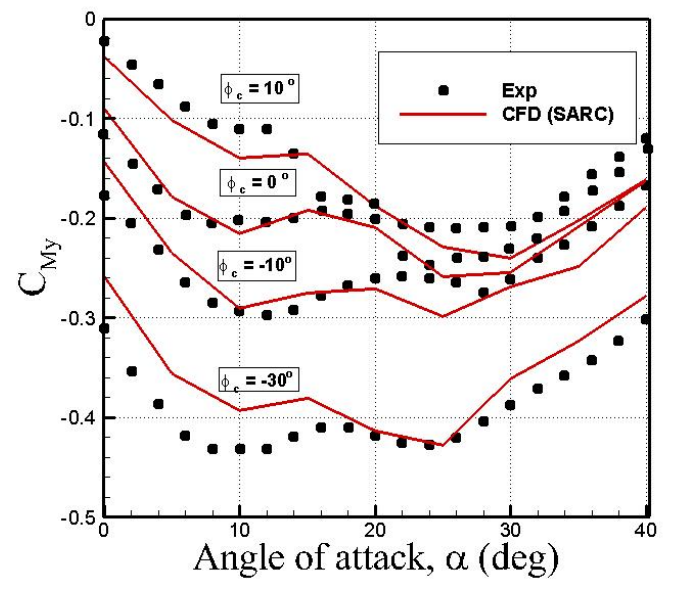

(b) pitch-moment coefficient

Figure 21: Validation of the overset mesh for canard deflections of $\phi_{c}=-10^{\circ}, 0^{\circ}, 10^{\circ}$, and $30^{\circ}$. 


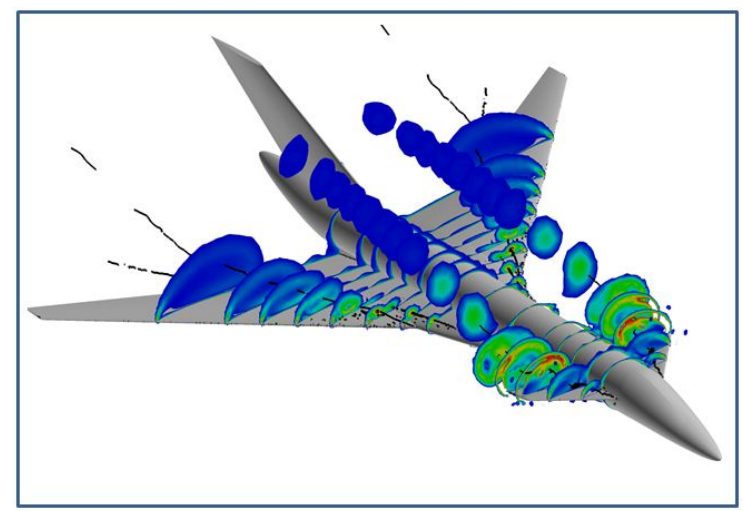

(a) $\phi_{c}=10^{\circ}$

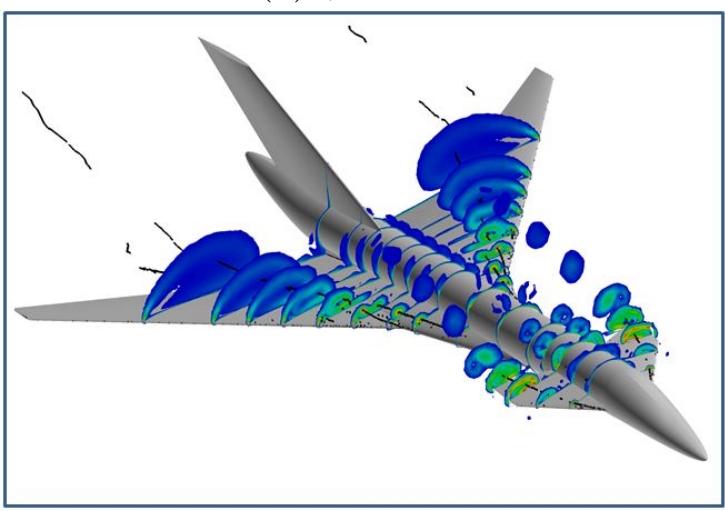

(c) $\phi_{c}=-10^{\circ}$

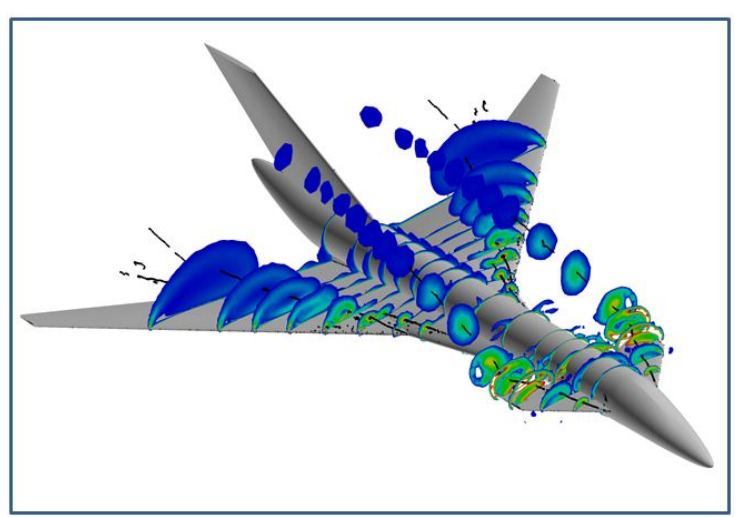

(b) $\phi_{c}=0^{\circ}$

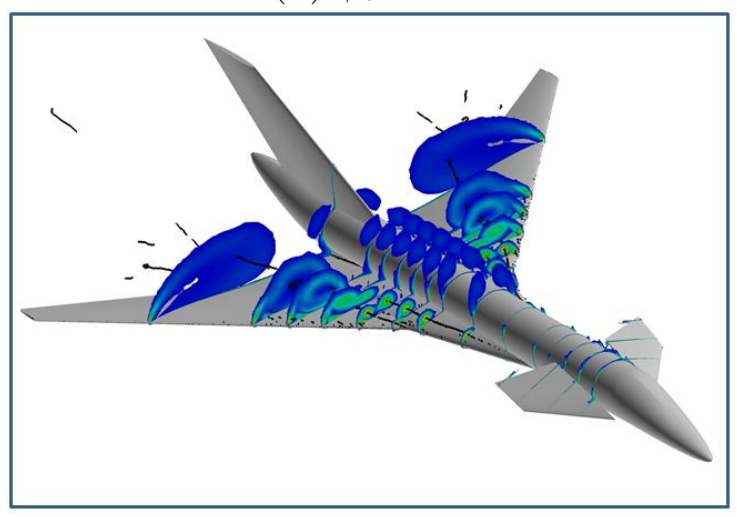

(d) $\phi_{c}=-30^{\circ}$

Figure 22: TCR flow solutions at $\alpha=20^{\circ}$ for different canard deflections. Iso-surfaces were created and colored by vorticity. The black lines show the core of vortices. 


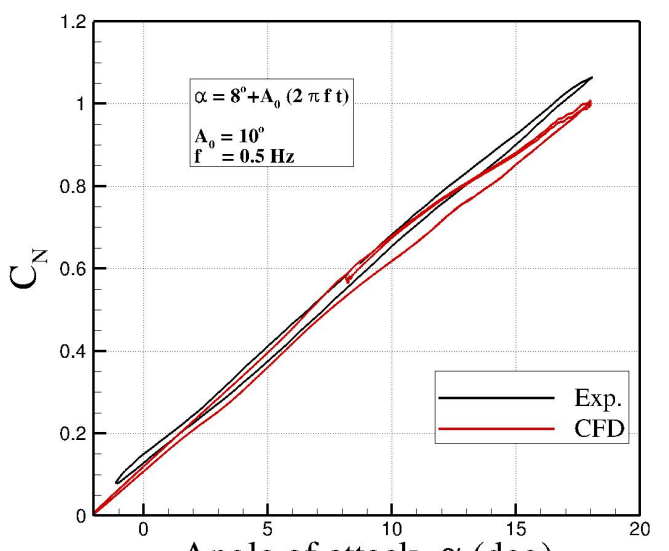

Angle of attack, $\alpha(\mathrm{deg})$
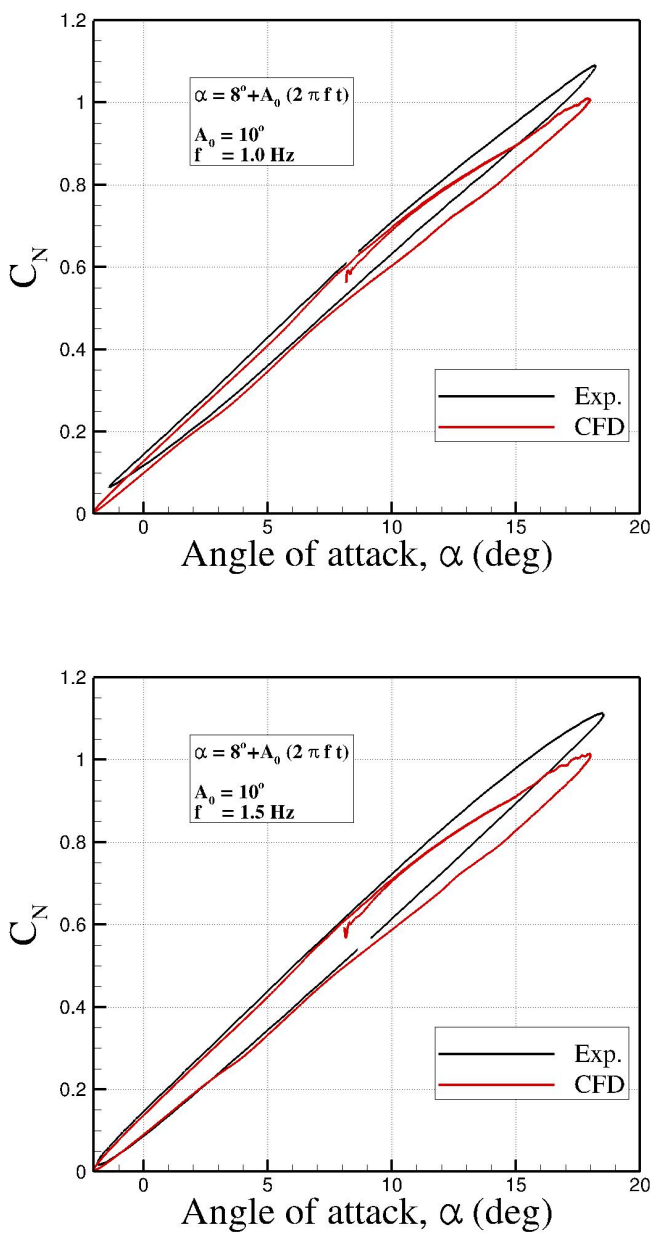

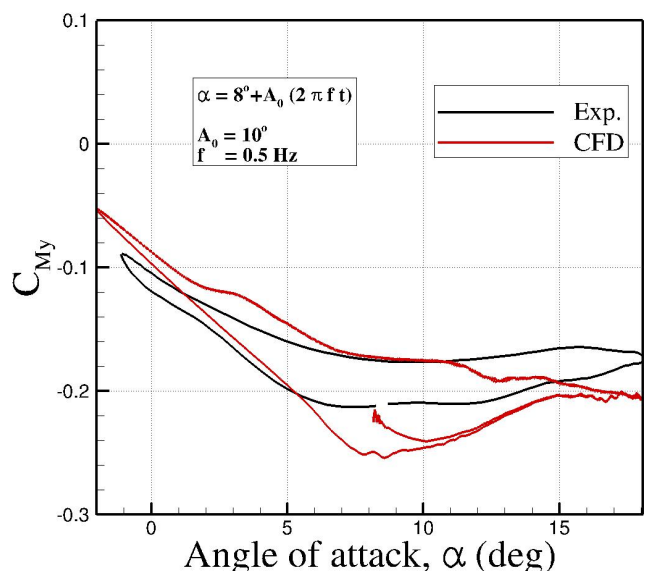

Angle of attack, $\alpha(\mathrm{deg})$
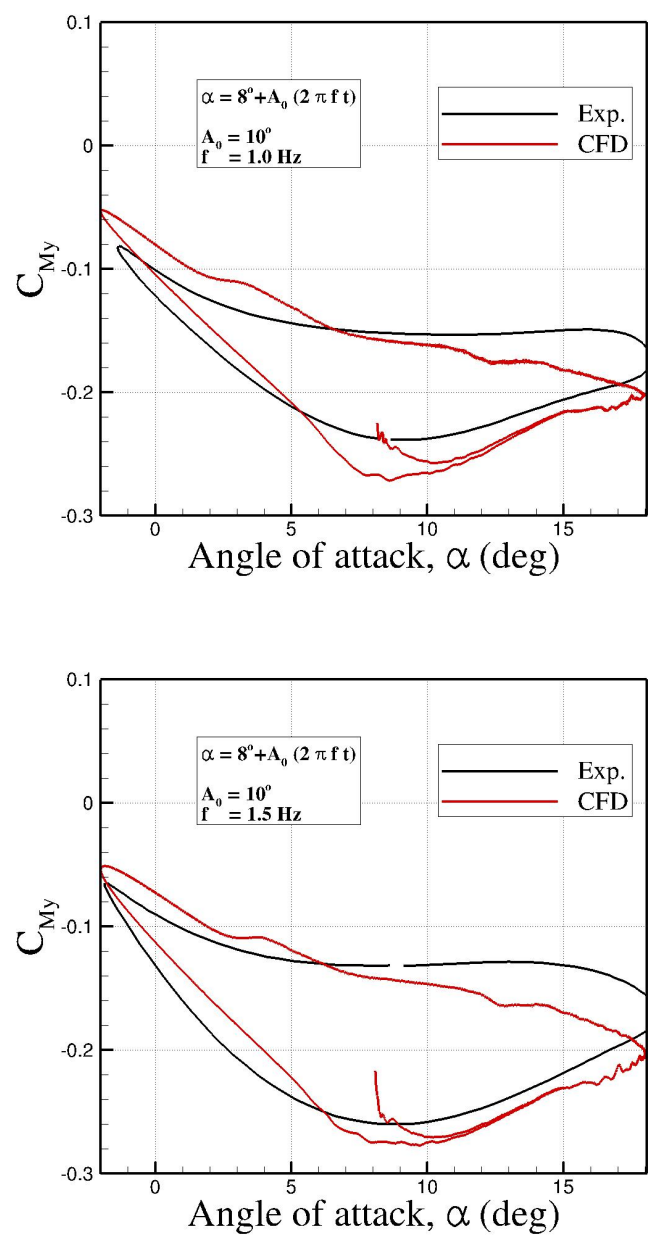

Figure 23: Validation of forced oscillation motions at $\phi_{c}=0^{\circ}$. The motions are defined as $\alpha=8^{\circ}+$ $10^{\circ} \sin (2 \pi f t)$ with $f=0.5,1.0$, and $1.5 \mathrm{~Hz}$. 


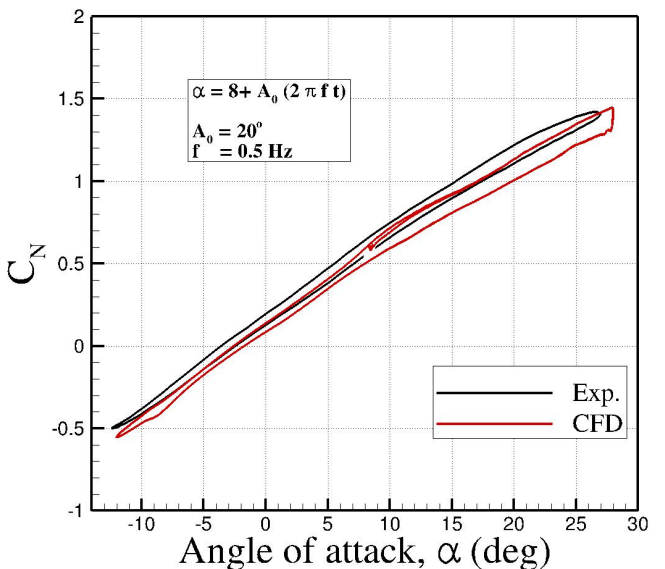

Angle of attack, $\alpha(\mathrm{deg})$
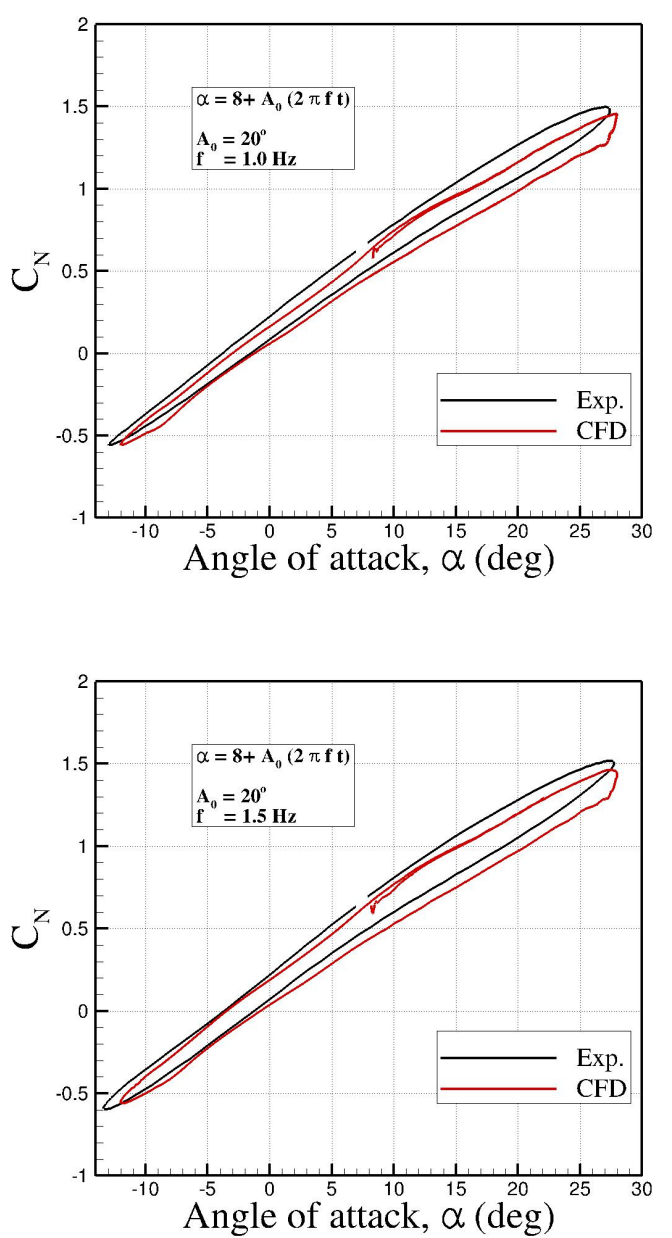
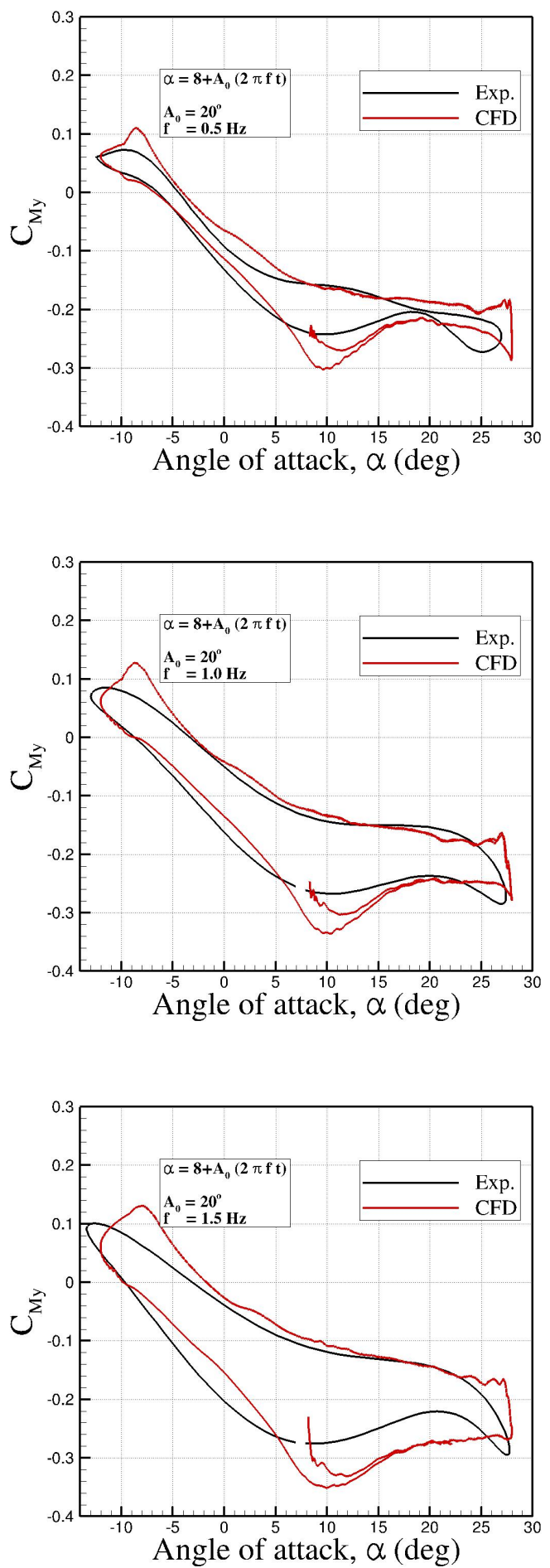

Figure 24: Validation of forced oscillation motions at $\phi_{c}=0^{\circ}$. The motions are defined as $\alpha=8^{\circ}+$ $20^{\circ} \sin (2 \pi f t)$ with $f=0.5,1.0$, and $1.5 \mathrm{~Hz}$. 

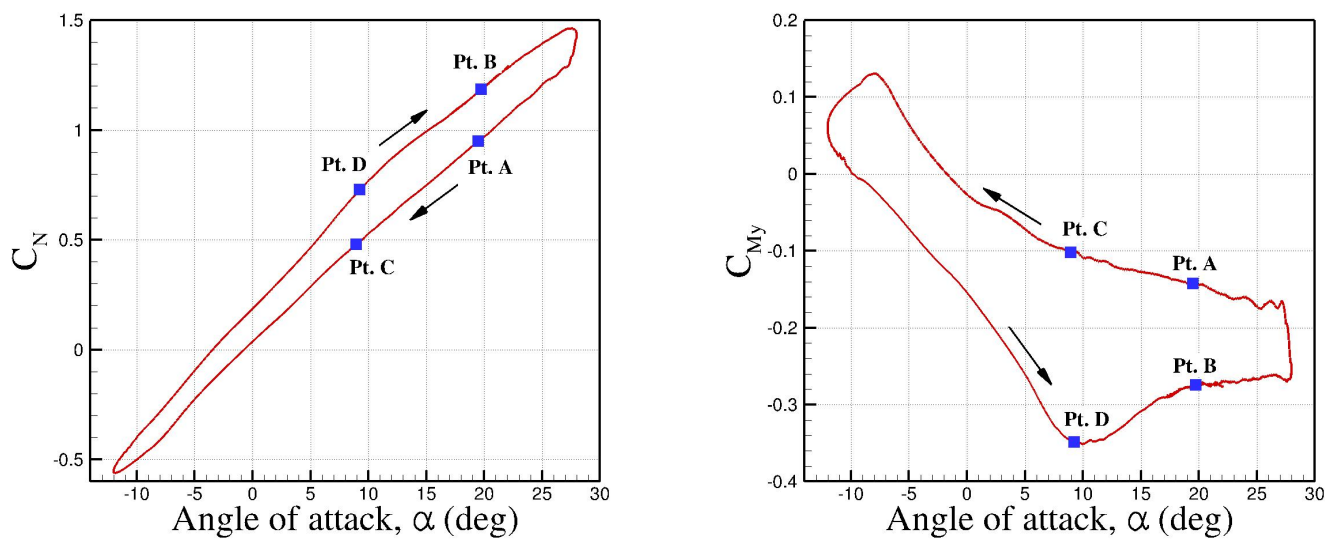

Pitching motion: $\alpha=8^{\circ}+20^{\circ} \sin (3 \pi t)$

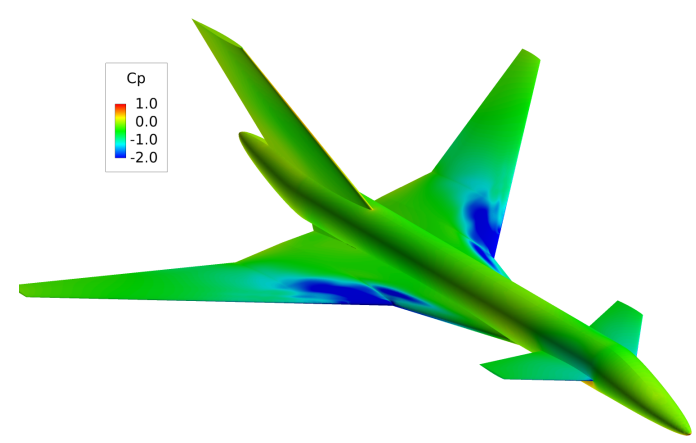

Point A. $\alpha=19.5^{\circ}$

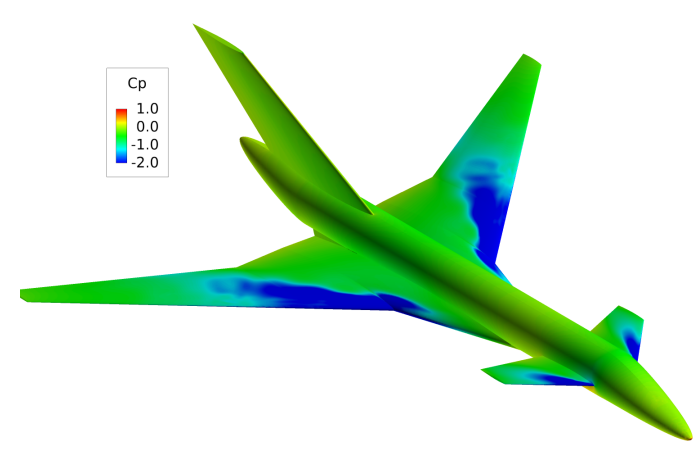

Point B. $\alpha=19.75^{\circ}$

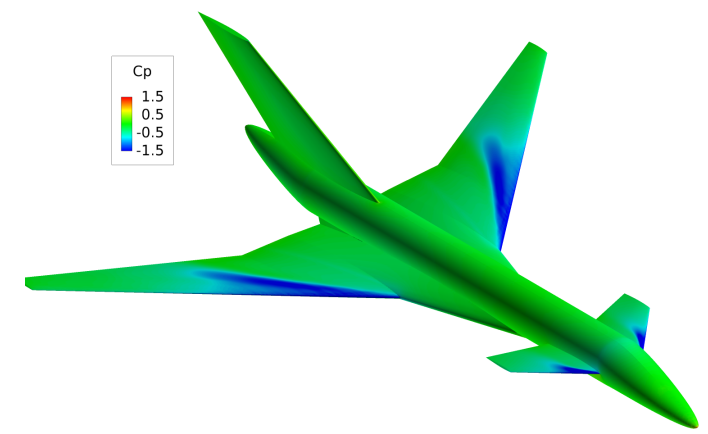

Point C. $\alpha=8.94^{\circ}$

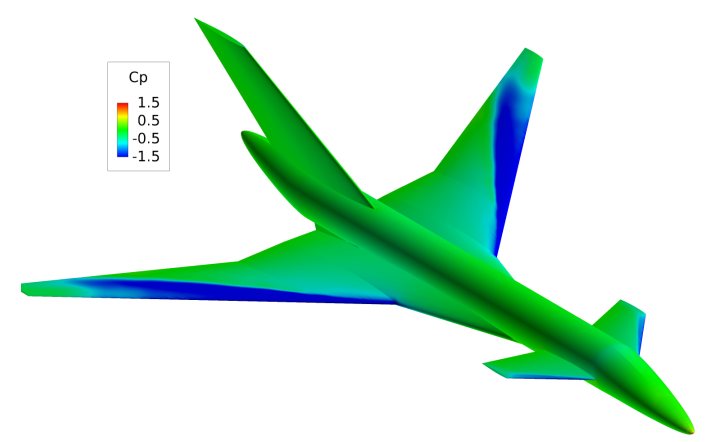

Point D. $\alpha=9.25^{\circ}$

Figure 25: TCR surface pressure solutions for a pitching motion simulation defined as $\alpha=8^{\circ}+20^{\circ} \sin (3 \pi t)$. 